\title{
CESR Conversion Damping Ring Studies of Electron Cloud Instabilities (CESR-TA)
}

\author{
Final Report \\ Grant Number: DE-FCO2-08ER41538 \\ Release Date: August 2, 2011
}

Principal Investigator: David L. Rubin

Project Director: Mark A. Palmer

Laboratory: $\quad$ Cornell University Laboratory for

Elementary-Particle Physics

Research carried out as part of the

international linear collider

Global Design Effort R\&D Program 


\title{
international linear collider
}

\begin{abstract}
In the International Linear Collider, two linear accelerators will accelerate bunches of positrons and electrons to over a hundred billion electron volts and collide them in a central detector. In order to obtain useful collision rates, the bunches, each containing twenty billion particles, must be compressed to a cross section of a few nanometers by a few hundred nanometers. In order to prepare these ultra high density bunches, damping rings (DRs) are employed before the linear accelerators. The DRs take the high emittance bunches that are provided by the electron and positron sources and, through the process of radiation damping, squeeze them into ultra low emittance beams that are ready for the main linear accelerators.
\end{abstract}

In the damping rings, a number of effects can prevent the successful preparation of the beams. In the electron ring, an effect known as the fast ion instability can lead to beam growth and, in the positron ring, the build-up of an electron cloud (EC), which interacts with the circulating bunches, can produce the same effect. EC build-up and the subsequent interaction of the cloud with the positron beam in the DR have been identified as major risks for the successful construction of a linear collider. The CESRTA research program at the Cornell Electron Storage Ring (CESR) was developed in order to study the build-up of the EC, the details of its impact on ultra low emittance beams, as well as methods to mitigate the impact of the cloud.

In the DR, the EC forms when synchrotron photons radiated from the circulating beam strike the walls of the vacuum chamber, resulting in the emission of photoelectrons. These low energy electrons can be accelerated across the vacuum chamber by the electric field of the beam, and strike the walls, causing the emission of secondary electrons. The secondary electrons are subsequently accelerated into the walls yet again via the same mechanism. The result is that the EC can rapidly begin to fill the vacuum chamber. In an electron DR, the EC build-up is limited by the Coulomb repulsion. But in a positron ring, the electrons are pulled into the potential well of the beam. The resulting interaction of the circulating bunches of positrons with the EC that ultimately limits DR performance.

Typically we store long trains of closely space bunches in the damping ring. The interaction of stored beam and the EC that is generated by the long train is manifested by different mechanisms:

1. The cloud focuses the beam, which causes a tune shift that increases along the bunch train as the cloud density increases.

2. The cloud electrons couple the motion of bunches along a train. Transverse motion of a leading bunch is transferred to the cloud, and subsequently to a trailing bunch which can result in a multibunch instability

3. The cloud couples the positrons in the head of the bunch to those in the tail of the same bunch, which can excite a "head-tail" instability.

4. The nonlinear fields of the EC can lead to emittance growth before the onset of instabilities.

The CESRTA collaboration, which includes researchers from Cornell University's Laboratory for Elementary-Particle Physics as well as more than 50 senior staff members from over a dozen accelerator laboratories and universities around the world, has operated CESR as a damping ring for the past three years to study these EC effects. A range of specialized instrumentation has been deployed to study the local build-up of the cloud in the vacuum chambers as well as the complicated dynamics exhibited when the beam and the EC interact. The program has significantly advanced our understanding of these issues and has helped identify the most promising methods to mitigate the impact of the EC on the DR beams. It has pointed the way towards a DR design that can meet the stringent specifications of the ILC. 


\section{international linear collider}

\section{Executive Summary}

The International Linear Collider is a machine designed to accelerate and collide bunches of electrons and positrons.at very high energies, from hundreds of billions to trillions of electron volts. These high energy collisions will yield states of matter that have not existed since the very early universe. Damping rings are an essential component of a linear collider. This grant has supported the investigation of the physics of linear collider damping rings.

In a linear accelerator, electrons and positrons are produced at low energy with diffuse position and momenta distributions. In order to ensure a significant annihilation rate when the distributions are accelerated and brought into collision at high energy, we cool the low energy bunches in damping rings, one for electrons and one for positrons. The cooled bunches that emerge from the damping rings have ultra low emittance and ultra high density. The phenomena that limit the achievable charge density and total beam current in the damping rings, and the development of techniques for extending that limit, are the subject of our investigations.

One of the most important limiting phenomena is the electron cloud (EC) effect. In the damping ring the circulating particles emit synchrotron radiation photons that strike the walls of the vacuum chamber, resulting in the emission of photoelectrons. The low energy electrons can be accelerated across the vacuum chamber by the electric field of the positron (or electron) beam and strike the walls, causing the emission of secondary electrons. The secondary electrons can subsequently be accelerated into the walls yet again by the same mechanism. The result is that an EC can begin to fill the chamber. In an electron damping ring, the EC is limited by the Coulomb repulsion. But in a positron ring, the electrons are trapped in the potential well of the beam.

It is the interaction of the circulating bunches of positrons with the EC that ultimately limits damping ring performance. Typically we store long trains of closely spaced bunches in the damping ring. We find that the density of the EC increases along the train. The bunches at the head of the train see few electrons while those near the tail pass through the cloud that is generated by all of the preceding bunches.

The interaction of stored beam and the EC is manifested by different mechanisms:

5. The cloud focuses bunches and we anticipate a tune shift along a bunch train that depends on the cloud density and distribution along the train.

6. The cloud electrons couple the motion of leading to trailing bunches. Transverse motion of a leading bunch is transferred to the cloud, and subsequently to a trailing bunch, which can result in the onset of a multi-bunch instability.

7. The cloud couples the head of the bunch to the tail of the same bunch, which can excite a synchro-betatron resonance.

8. Incoherent, sub-threshold emittance growth, in which the emittance is observed to increase even when the cloud density is below the threshold for synchro-betatron, or head-tail, instability may also be present.

All of these mechanisms have been observed and quantified over the course of our investigations. These observations are being used to improve the physics models describing the phenomena. We have achieved a level of understanding of the electron cloud effect that allows for the design of a damping ring that will perform at the required level with a high degree of confidence.

Along with our study of the interaction of the electron cloud and the circulating beam, we have investigated techniques for mitigating the growth of the cloud. In particular, we have tested vacuum chamber coatings, and geometries that reduce the secondary electron yield. We measure the local cloud 


\section{international linear collider}

density using retarding field analyzers (RFAs). Dozens of retarding field analyzers were installed around the ring in order to monitor cloud growth in different magnetic fields, both in regions characteristic of the CESR vacuum chambers as well as in vacuum chambers incorporating EC mitigations.

In order to conduct the above studies, it was necessary to reconfigure the Cornell Electron Storage Ring (CESR) as a low emittance damping ring. This required a redesign of the storage ring focusing lattice and redeployment of superconducting damping wigglers. The ring was instrumented with high precision, high bandwidth beam position monitors and x-ray beam size monitors for characterization of both electron and positron beams. Measurements with both species provided a unique capability for distinguishing speciesdependent effects. Characterization of EC effects in the ultra low emittance regime required a parallel program to understand, identify, and correct sources of emittance dilution. By the conclusion of the grant period, we developed tuning techniques that routinely allowed us to achieve $<10 \mathrm{pm}$-rad vertical emittance. The findings of the R\&D program have been applied to the ILC damping ring design.

It is important to note that the CESRTA collaboration has involved over 50 senior staff from more than a dozen international laboratories and universities. The contributions of this talented team of researchers have been essential to the success of the research program. 


\section{international linear collider}

\section{Summary of Project Milestones and Deliverables}

The principal elements specified for the CESRTA R\&D Program during the period of this grant were:

1. Electron cloud (EC) growth and instability studies:

a. Characterize cloud growth in wiggler, dipole, and quadrupole fields as well as drift regions, with a particular emphasis on the measurements in the magnetic field regions

b. Test the effectiveness of various electron cloud suppression techniques

c. Measure instability thresholds and emittance growth at the lowest achievable emittance

d. Test instrumented wiggler vacuum chambers at $5 \mathrm{GeV}$, the specified ILC damping ring operating energy

e. Provide inputs to the ILC damping ring design based on these studies

2. Development of low emittance tuning techniques with a goal of achieving $<20 \mathrm{pm}$-rad vertical emittance before the conclusion of the funding period:

a. Implement high resolution single turn beam position monitor (BPM) electronics for precision measurement of orbit and dispersion

b. Upgrade survey and alignment hardware to improve the efficiency and accuracy of positioning guide field magnets

c. Analyze beam based measurements to identify sources of emittance dilution

d. Implement corrective measures based on findings (c)

e. Develop and test low emittance tuning algorithms

3. Development of a high resolution X-ray beam size monitor (xBSM) to measure ultra low emittance beams:

a. Develop a high resolution 1-dimensional x-ray monitor capable of measuring the micronscale vertical beam sizes associated with the ultra low emittance beams

b. Develop a detector capable of making single-pass measurements of individual bunches in a bunch train on a turn-by-turn basis.

Table 1 provides a detailed overview of the quarter-by-quarter milestones and deliverables that were put in place to achieve these goals. The core experimental effort was targeted to conclude by the end of FY10 in order to provide timely inputs for the ILC damping ring technical design. With the strong support of ILC management and the CESRTA Collaboration, this goal was successfully accomplished. The following list highlights some of the key experimental and reporting milestones that were achieved during this period:

- May 2008 - Start of dedicated experimental program;

- October 2008 - CESR stored the first beam after being reconfigured as a damping ring;

- February 2009 - Initial low emittance operations were established and the positron x-ray beam size monitor (xBSM) measurements of the vertical beam size were found consistent with a vertical emittance $\varepsilon_{\mathrm{y}}<40$ pm-rad;

- July 2009 - Completed the last major "CESR Conversion" down. At this point all electron cloud experimental areas and the beam instrumentation infrastructure for the low emittance instrumentation were in place, although not all systems were fully operational;

- December 2009 - First operations with a fully digital BPM system; measurements of the vertical emittance indicated $\varepsilon_{\mathrm{y}} \sim 31 \mathrm{pm}$-rad;

- May 2010 - All major EC mitigation tests for Phase I of the program deployed; vertical emittance target of $\varepsilon_{\mathrm{y}}<20$ pm-rad achieved; techniques for measuring single bunch EC-induced instabilities (head-tail instabilities) fully operational;

- October 2010 - Nominal conclusion of Phase I of the experimental program - 240 experimental days provided; CESRTA EC results incorporated into the ILC positron damping ring EC mitigation plan; EC dynamics studies providing key inputs to projections of ILC DR performance expectations. 


\section{international linear collider}

Table 1: Summary of Detailed Project Deliverables/Milestones as specified for the ILC Americas Regional Team. The initial target quarter and the date of actual delivery are noted.

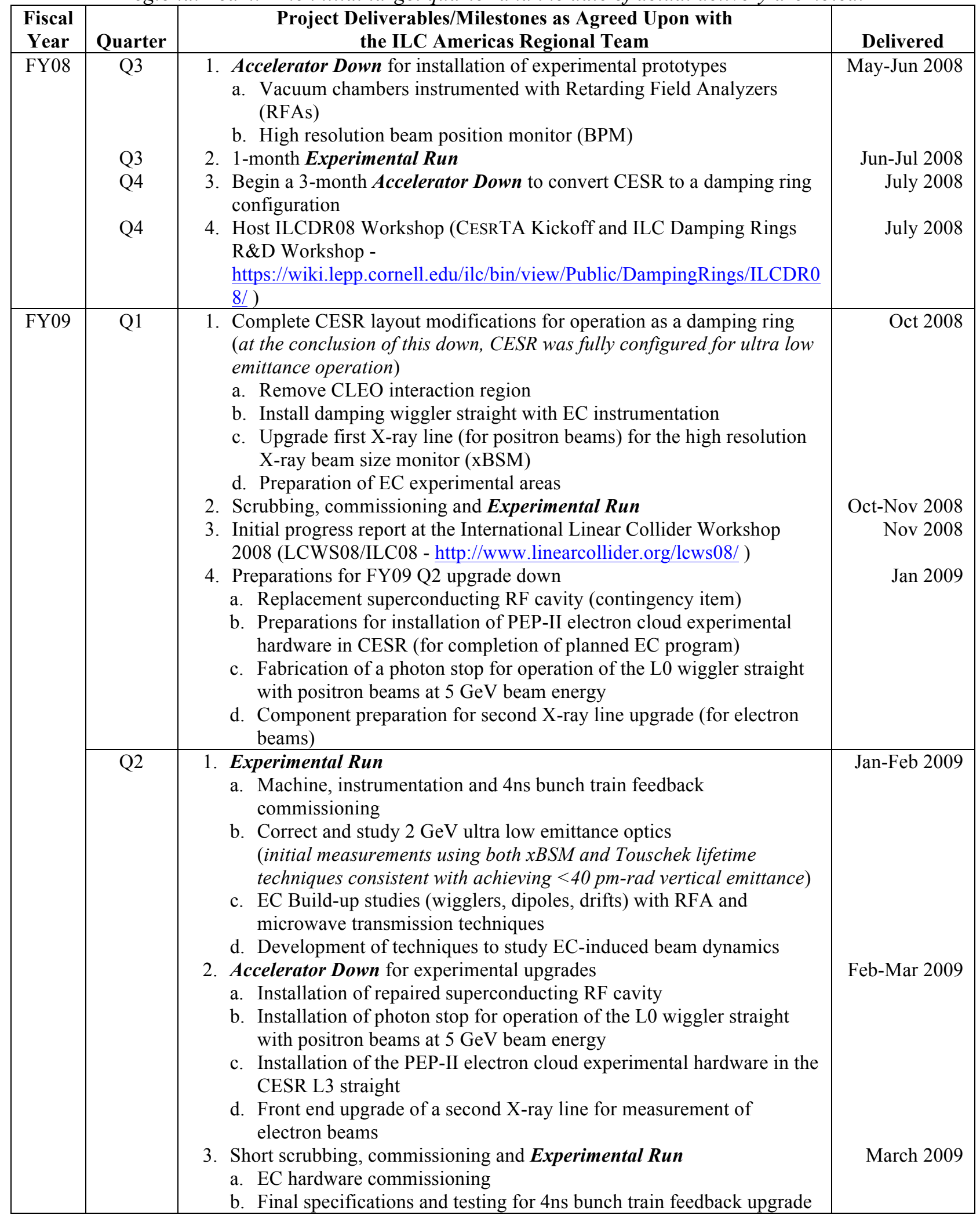




\section{international linear collider}

\begin{tabular}{|c|c|c|c|}
\hline $\begin{array}{c}\text { Fiscal } \\
\text { Year }\end{array}$ & Quarter & $\begin{array}{c}\text { Project Deliverables/Milestones as Agreed Upon with } \\
\text { the ILC Americas Regional Team }\end{array}$ & Delivered \\
\hline \multirow[t]{2}{*}{ FY09 } & Q3 & $\begin{array}{l}\text { 1. Progress report to the ILC Accelerator Advisory Panel at the Joint } \\
\text { ACFA Physics and Detector Workshop and GDE Meeting on the } \\
\text { International Linear Collider (TILC09 - http://tilc09.kek.jp/) } \\
\text { 2. Commissioning and Experimental Run (at the conclusion of this run } \\
\text { approximately 45\% of the specified } 240 \text { CESRTA operations days had } \\
\text { been provided) } \\
\text { a. Instrumentation and feedback commissioning } \\
\text { b. Low emittance tuning } \\
\text { c. EC measurements } \\
\text { 3. Conduct final major Accelerator Down for the CESR Conversion to the } \\
\text { CESRTA configuration } \\
\text { a. Deployment of vacuum chambers with EC diagnostics and } \\
\text { mitigations (at this point experimental chambers were deployed in } \\
\text { drift, dipole, wiggler, and quadrupole regions) } \\
\text { b. Continued deployment of upgraded beam instrumentation (BPMs, } \\
\text { xBSM - note: the schedule for full BPM system deployment was } \\
\text { extended into the first half of FY10) } \\
\text { c. Complete 4ns feedback upgrade } \\
\text { 4. Host CTA09 Workshop to evaluate project progress, prioritize electron } \\
\text { cloud research topics, and coordinate program with collaborators - } \\
\text { https://wiki.lepp.cornell.edu/ilc/bin/view/Public/DampingRings/CTA09 } \\
\text { /WebHome ) }\end{array}$ & May-Jun 2009 \\
\hline & Q4 & $\begin{array}{l}\text { 1. Conduct Commissioning and Experimental Run } \\
\text { a. Commission upgraded BPM system (90\% of ring operational with } \\
\text { digital BPM readout modules by conclusion of this run) } \\
\text { b. Experiments targeted at taking full advantage of ring upgraded } \\
\text { instrumentation } \\
\text { 2. Prepare detailed evaluation of the operating parameters, measurements, } \\
\text { and data-simulation comparisons for operating CESR in the damping } \\
\text { ring configuration. Evaluations presented at the } 2009 \text { Linear Collider } \\
\text { Workshop of the Americas (LCWA09 - } \\
\text { http://panda.unm.edu/LCWA09/) } \\
\text { 3. Prepare a detailed electron cloud experimental plan for the } 3 \\
\text { experimental runs planned for FY10 - these plans were developed as } \\
\text { part of the CTA09 Workshop (see above) }\end{array}$ & June 2009 \\
\hline \multirow[t]{2}{*}{ FY10 } & Q1 & $\begin{array}{l}\text { 1. Complete switchover to fully digital BPM system } \\
\text { 2. Commissioning and Refinement of xBSM systems for measuring both } \\
\text { electron and positron beams } \\
\text { 3. Conduct Experimental Run ( } 70 \% \text { of the planned CESRTA operations } \\
\text { days delivered at the conclusion of this run) } \\
\text { a. Low emittance tuning (during this run achieved } \varepsilon_{y} \sim 31 \text { pm-rad) } \\
\text { b. EC mitigation studies } \\
\text { c. EC beam dynamics studies }\end{array}$ & $\begin{array}{r}\text { Dec } 2009 \\
\text { Nov-Dec } 2009 \\
\text { Nov-Dec } 2009\end{array}$ \\
\hline & Q2 & $\begin{array}{l}\text { 1. Accelerator Down for installation of vacuum chambers to test EC } \\
\text { mitigations } \\
\text { a. Wiggler chamber with clearing electrode (CU-KEK-LBNL-SLAC } \\
\text { collaboration) } \\
\text { b. Quadrupole chamber with TiN coating } \\
\text { c. Drift chambers with NEG and amorphous carbon (CERN) coatings } \\
\text { 2. Carry out first in situ SEY station measurements to study beam } \\
\text { processing effects on surfaces during CHESS operations } \\
\text { 3. Provide a program status report at the ILC10 Workshop } \\
\text { (http://lcws10.ihep.ac.cn/) }\end{array}$ & $\begin{array}{l}\text { Jan-Mar } 2010 \\
\text { Mar-Apr } 2010\end{array}$ \\
\hline
\end{tabular}




\section{international linear collider}

\begin{tabular}{|c|c|c|c|}
\hline $\begin{array}{l}\text { Fiscal } \\
\text { Year }\end{array}$ & Quarter & $\begin{array}{c}\text { Project Deliverables/Milestones as Agreed Upon with } \\
\text { the ILC Americas Regional Team }\end{array}$ & Delivered \\
\hline \multirow[t]{2}{*}{ FY10 } & Q3 & $\begin{array}{l}\text { 1. Conduct Experimental Run } \\
\text { a. Low emittance tuning } \\
\text { (At the conclusion of this run, operation with } \varepsilon_{\mathrm{y}}<20 \text { pm-rad had } \\
\text { been achieved) } \\
\text { b. Instrumentation development } \\
\text { c. EC build-up and mitigation studies } \\
\text { d. EC-induced beam dynamics studies }\end{array}$ & May 2010 \\
\hline & Q4 & $\begin{array}{l}\text { 1. Accelerator Down for installation of vacuum chambers for any } \\
\text { remaining high priority EC mitigation tests } \\
\text { 2. Conduct Experimental Run } \\
\text { (at conclusion of this experimental period, 240 CESRTA operations } \\
\text { days had been provided as part of the research program) } \\
\text { a. Low emittance tuning targeting demonstration of final vertical } \\
\text { emittance goal (already demonstrated in preceding run) } \\
\text { b. EC build-up and mitigation studies } \\
\text { c. EC-induced beam dynamics studies }\end{array}$ & $\begin{array}{r}\text { Aug } 2010 \\
\text { Split in } 2 \text { parts: } \\
\text { Jul-Aug } 2010 \\
\text { Sept } 2010\end{array}$ \\
\hline \multirow[t]{2}{*}{ FY11 } & Q1 & $\begin{array}{l}\text { 1. Host ECLOUD10 Workshop } \\
\text { (http://www.lepp.cornell.edu/Events/ECLOUD10/) } \\
\text { a. Report on CESRTA Phase I Results } \\
\text { b. Incorporate CESRTA Phase I Results into an EC mitigation } \\
\text { recommendation for the ILC positron damping ring } \\
\text { 2. Provide a summary of Phase I of the CESRTA R\&D Program at } \\
\text { IWLC2010 (https://espace.cern.ch/LC2010/default.aspx) } \\
\text { 3. Conduct short Experimental Run to follow up on results obtained in } \\
\text { Phase I of the program } \\
\text { a. Low emittance tuning } \\
\text { (At the conclusion of this run, operation with } \varepsilon_{\mathrm{y}}<10 \mathrm{pm} \text { had been } \\
\text { achieved) } \\
\text { b. EC build-up and mitigation studies } \\
\text { c. EC-induced beam dynamics studies } \\
\text { 4. Begin preparation of a CESRTA Phase I Report (targeted for release in } \\
\text { the last half of FY11) }\end{array}$ & $\begin{array}{l}\text { Oct } 2010 \\
\text { Dec } 2010\end{array}$ \\
\hline & Q2 & $\begin{array}{l}\text { 1. Support design studies and documentation required for the } 2^{\text {nd }} \text { ILC } \\
\text { Baseline Assessment Workshop } \\
\text { 2. Participate in planning and studies needed for the ILC DR lattice } \\
\text { choice (lattice performance issues delayed the final lattice down-select } \\
\text { from March to June 2011) } \\
\text { 3. Continue preparation of the CESRTA Phase I Report for release in late } \\
\text { FY11 }\end{array}$ & $\begin{array}{r}\text { Jan } 2011 \\
\text { June } 2011\end{array}$ \\
\hline
\end{tabular}




\section{international linear collider}

\section{Project Summary}

\subsection{Introduction}

One of the principal R\&D issues for the positron damping ring (DR) of the International Linear Collider (ILC) is to ensure that the build-up of the electron cloud (EC) in the vacuum chambers can be kept below the levels at which EC-induced emittance growth and beam instabilities occur. During Part I (2008-2010) of the ILC Technical Design Phase (TDP) a focused effort to study methods of suppressing the EC as well as measuring its impact on ultra low emittance beams was undertaken at the Cornell ElectronPositron Ring Test Accelerator (CESRTA). Work has also been underway at various laboratories around the world to develop better techniques to mitigate the build-up of the EC. We describe the research effort that has been carried out at Cornell University with the CESRTA collaboration [1,2] and jointly supported by this grant and a companion grant (PHY-0734867) from the US National Science Foundation. In close coordination with researchers from the other laboratories, a major emphasis has been placed on developing and benchmarking simulation tools as well as measurement techniques. In October 2010, the ECLOUD10 Workshop was held at Cornell University [3] providing a venue to review the status of our understanding of the EC.

In order to incorporate the research results into the ILC DR design, an ECLOUD Working Group has been formed whose main objective is to provide recommendations on the EC mitigation techniques to apply to the DR design, based on the results of the R\&D program $[4,5]$. This objective has recently been achieved in a dedicated Working Group meeting [6] during the ECLOUD10 workshop, with a significant level of participation by the experts attending the workshop. The preliminary recommendations are summarized in section 3.4.5.

The CESRTA research program was approved in late 2007 to carry out electron cloud R\&D in support of the ILC technical design. The first dedicated experiments using the Cornell Electron-Positron Storage Ring (CESR) began in March 2008 at the conclusion of 28 years of colliding beam operations for the CLEO experiment [7]. Two principal goals were specified for the program. The first was to characterize the build-up of the EC in each of the key magnetic field regions of the accelerator, particularly in the dipoles and wigglers, and to study the most effective methods of suppressing it in each of these regions. This required the design and installation of detectors to study the local build-up of the cloud in each of these environments as well as a supporting simulation program to fully characterize and understand the results. The second goal was to study the impact of the EC on ultra low emittance beams. The ILC DR design targets a geometric vertical emittance of $2 \mathrm{pm}$-rad and no positron ring has been operated in this emittance regime. By benchmarking EC instability and emittance growth simulations in a regime closer to that specified for the DR, confidence in our projections of the final DR performance can be significantly improved. This in turn determines whether further R\&D will be required to achieve the necessary design specifications. In order to carry out these measurements, CESR had to be reconfigured as a damping ring and upgraded with the necessary beam instrumentation for low emittance optics correction and characterization of the resulting beams.

\subsection{The CESR Conversion}

Modification of CESR into a damping ring configuration involved three main thrusts:

- Relocation of 6 of the 12 CESR-c damping wigglers [8,9] to the L0 straight to enable ultra low emittance CESRTA operation [7];

- Upgraded beam instrumentation to achieve and characterize ultra low emittance beams. This included deployment of a high resolution BPM system [10] and x-ray beam size monitors (xBSMs) for both positron and electron beams [11];

- Addition of vacuum system diagnostics for characterization of local EC growth in a range of vacuum chambers. Local EC diagnostics include retarding field analyzers (RFAs) $[12,13]$, TE wave transmission hardware [14], and shielded pickups for time-resolved measurements [15]. 


\section{international linear collider}

Table 2 shows the CESRTA lattice parameters for operation at 2 and $5 \mathrm{GeV}$. At $2 \mathrm{GeV}, 90 \%$ of the synchrotron radiation power is provided by the 12 damping wigglers and a horizontal emittance of $2.6 \mathrm{~nm}$-rad is obtained [16]. During phase I of the CESRTA program, a vertical emittance target of $<20$ pm-rad (10× the ILC DR vertical emittance target) was specified. A key element of the R\&D program is the flexibility of CESR operation. CESR allows operation between 1.8 and $5.3 \mathrm{GeV}$ with both positron and electron beams. The ability to operate over a wide range of energies, bunch spacings and bunch intensities enables systematic probes of primary photoelectron and secondary electron contributions to EC build-up in the vacuum chambers which are not feasible at any other facility.

A novel element of the CESRTA upgrade has been the development of a high resolution x-ray beam size monitor (xBSM) capable of single pass measurements of each bunch in a train. Figure 1 shows one of the InGaAs detectors wire-bonded to its circuit board along with a single-pass fit of data acquired using pinhole imaging with a $1 \mathrm{~mA}$ positron bunch $\left(1.6 \times 10^{10}\right.$ particles/bunch). In addition to pinhole imaging, coded aperture and Fresnel zone plate optics have also been installed in both the positron and electron beam lines. These detectors represent our principal tool for verifying the vertical beam size in the ultra low emittance machine optics.

Table 2: $2 \mathrm{GeV}$ and $5 \mathrm{GeV}$ lattice parameters for CESRTA.

\begin{tabular}{|c|c|c|}
\hline Energy [GeV] & 2.085 & 5.0 \\
\hline No. Wigglers & 12 & 6 \\
\hline Wiggler Field [T] & 1.9 & 1.9 \\
\hline$Q_{x}$ & \multicolumn{2}{|c|}{14.57} \\
\hline$Q_{y}$ & \multicolumn{2}{|c|}{9.6} \\
\hline$Q_{z}$ & 0.075 & 0.043 \\
\hline$V_{R F}[M V]$ & 8.1 & 8 \\
\hline$\varepsilon_{x}[n m-r a d]$ & 2.6 & 35 \\
\hline$\tau_{x, y}[m s]$ & 57 & 20 \\
\hline$\alpha_{p}$ & $6.76 \times 10^{-3}$ & $6.23 \times 10^{-3}$ \\
\hline$\sigma_{l}[\mathrm{~mm}]$ & 9.2 & 15.6 \\
\hline$\sigma_{E} / E[\%]$ & 0.81 & 0.93 \\
\hline$t_{b}[n s]$ & \multicolumn{2}{|c|}{$\geq 4$, steps of 2} \\
\hline
\end{tabular}
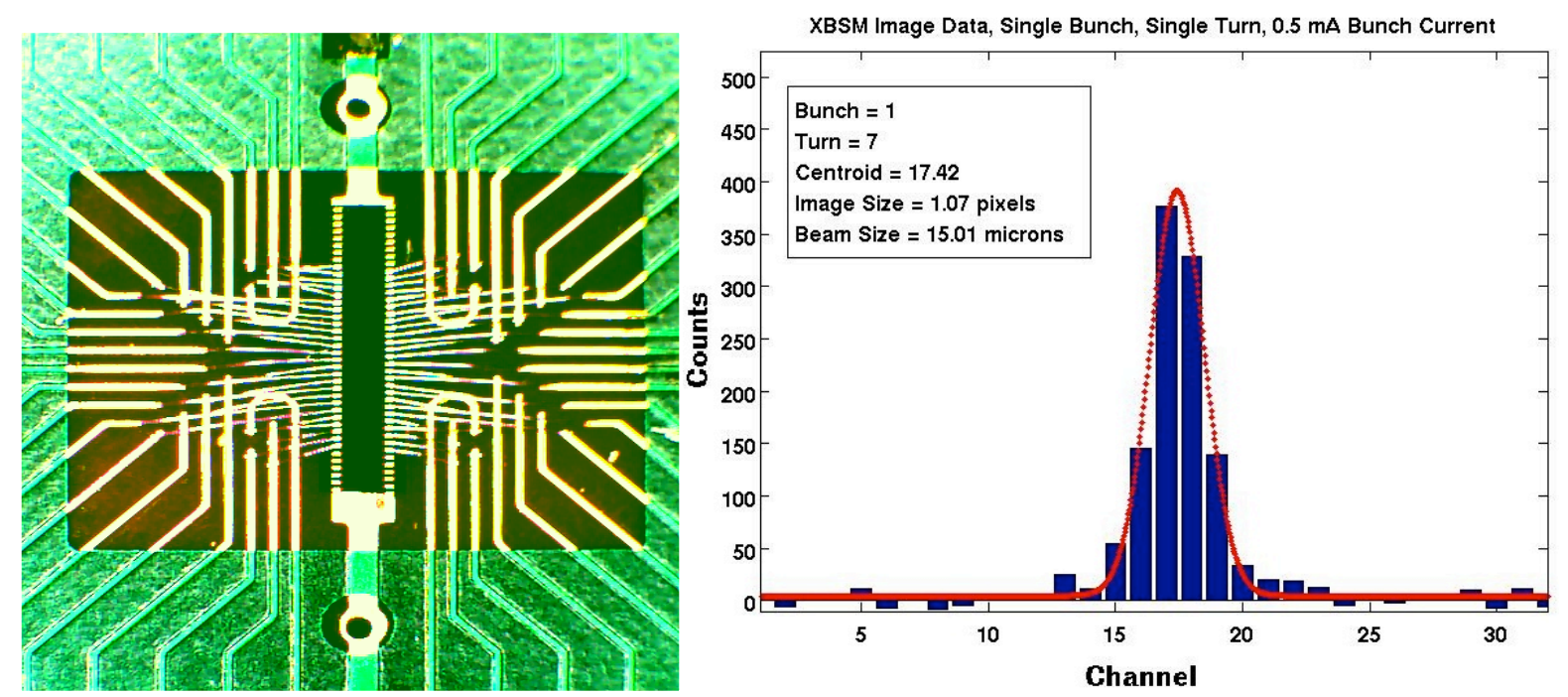

Figure 1: The left image shows an XBSM detector, an InGaAs diode array, mounted on its circuit board. 32 diodes of $400 \mu \mathrm{m}$ width and $50 \mu \mathrm{m}$ pitch are utilized in each detector. The plot on the right shows a single turn fit to data acquired from a bunch with $0.8 \times 10^{10}$ particles (at $2.1 \mathrm{GeV}$ beam energy) using a heavy metal slit as the $x$-ray imaging optic. 


\section{international linear collider}

Figure 2 shows the layout of the L0 straight after installation of the 6-wiggler string. This region represents one of four dedicated CESRTA EC experimental areas. It is equipped with extensive diagnostics to study the growth and mitigation of the EC in wigglers. A second EC experimental region was installed on the opposite side of CESR in the L3 straight. Figure 3 shows the layout of the L3 region. It supports 4 EC experiments: a large bore quadrupole housing a test chamber; the PEP II chicane, for dipole chamber tests, which was relocated from SLAC after the early termination of PEP II operations; a drift (field free region) chamber test section presently configured for testing TiZrV (NEG) test chambers; and an in-situ SEY measurement station which supports studies of the processing rates and equilibrium SEY properties of various technical surfaces. In addition to the L0 and L3 experimental regions, two arc sections were configured for flexible installation of experimental drift chambers to study the performance of various mitigations in the photon environment of the CESR arcs.

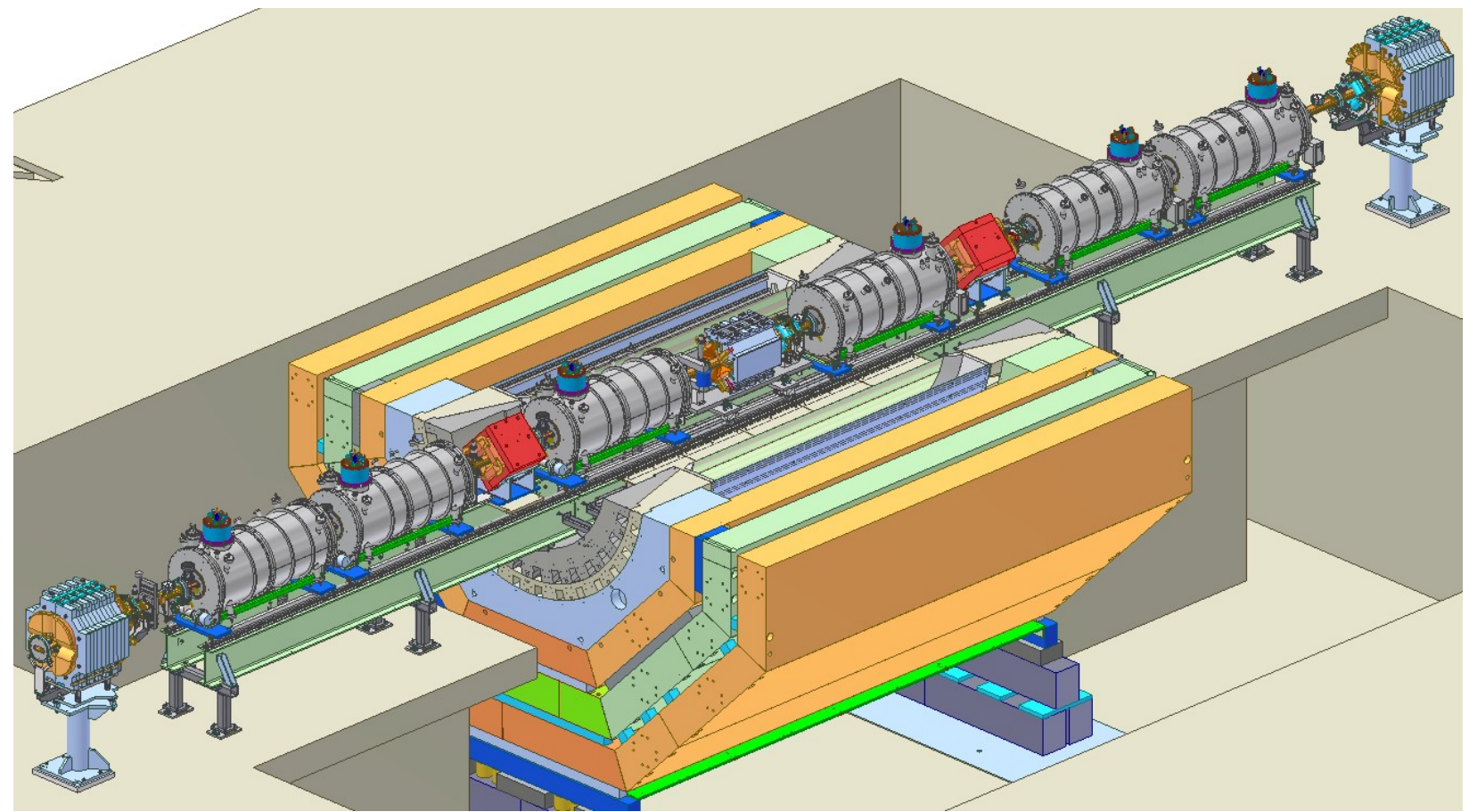

Figure 2: Layout of the CESR LO wiggler straight and EC experimental region with a cutaway view of the CLEO detector. 6 superconducting CESR-c type wigglers are deployed in the straight, which is configured for zero dispersion operation. The straight includes extensive vacuum diagnostics: RFAs, residual gas analyzer, and TE wave measurement hardware. 


\section{international linear collider}

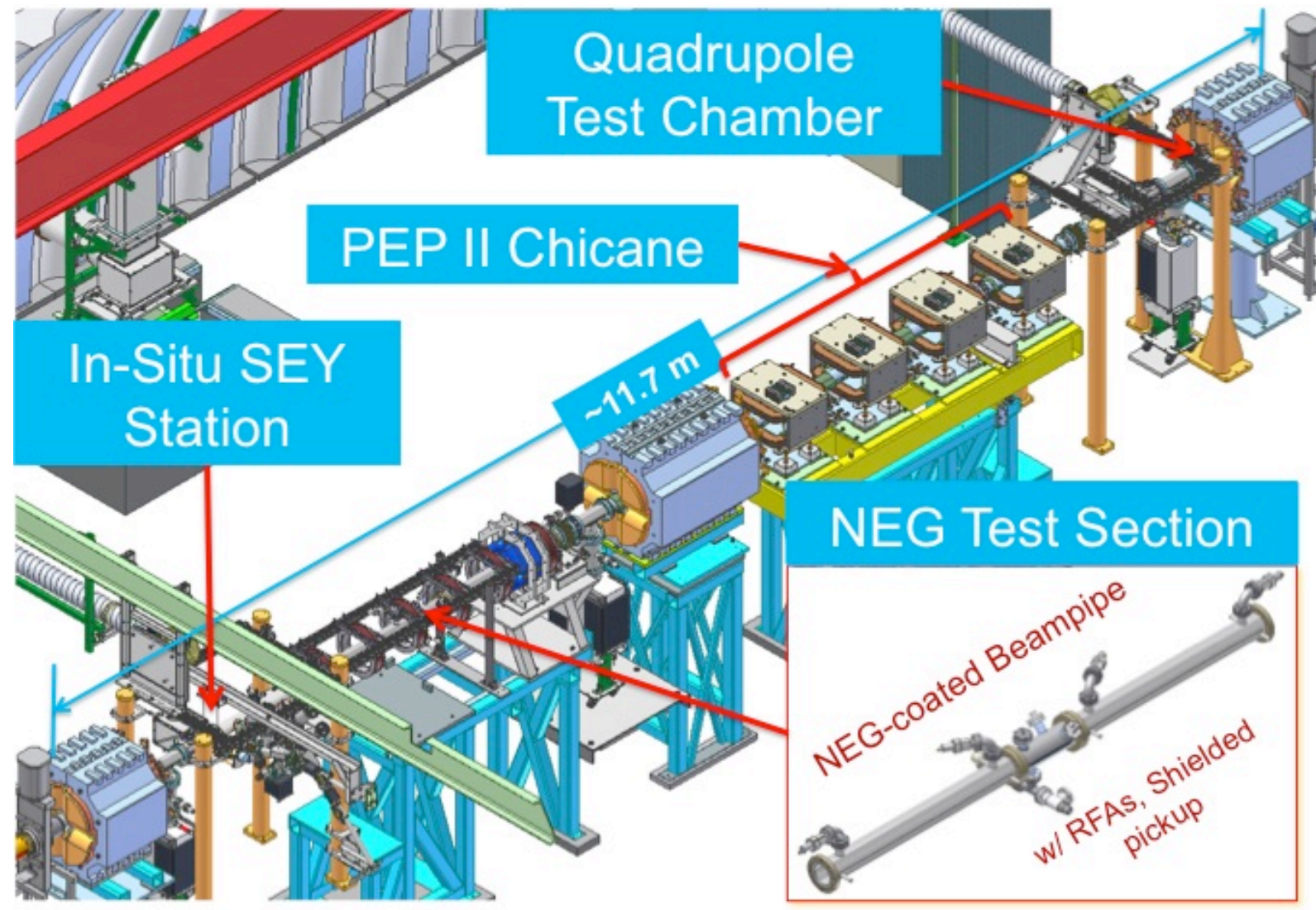

Figure 3: Layout of the CESR L3 straight and EC experimental region. Tests of EC mitigations in drift, dipole and quadrupole chambers are possible in this region. Additionally, an in-situ SEY station is also installed which allows characterization of the rate of processing and equilibrium SEY properties of various vacuum system technical surfaces.

\subsection{Low Emittance Tuning R\&D at CESRTA}

\subsubsection{Introduction}

As described above, an essential component of the electron cloud program is to measure the cloud density threshold for emittance growth. Sensitivity to emittance growth depends on first establishing the lowest possible emittance in the zero current limit. The electron cloud research also seeks to identify EC-induced beam instabilities. Insofar as the beam-cloud interaction depends on the beam charge density, it is again important to measure thresholds with a low emittance beam. The program to achieve low emittance included: design and implementation of low emittance storage ring optics, calculations via modeling of sensitivities of vertical emittance to various emittance diluting magnet misalignments; survey and alignment of guide field dipoles and quadrupoles at the level indicated by the sensitivity calculations; implementation of precision beam position monitors for identifying misalignments through beam based measurements; development of an x-ray beam size monitor for measuring the very small beam sizes, and development of low emittance tuning techniques and associated fitting algorithms to correct orbit, optics, and dispersion errors.

\subsubsection{Low Emittance Optics}

As noted above the layout of the storage ring was configured during the summer of 2008 for low emittance operation [1,7]. Six of the twelve damping wigglers were moved from the machine arcs to the $18 \mathrm{~m}$ (L0) straight that became available with the removal of the CLEO HEP detector $[8,9]$. The CLEO low beta final focus insert was replaced with standard FODO optics. The vertical separators were removed from the diametrically opposite straight (L3) to reduce longitudinal impedance and make space 


\section{international linear collider}

for instrumentation. The magnetic optics for damping ring studies were all designed with zero horizontal dispersion in the L0 wiggler straight, and in the arc wiggler straights in order to minimize horizontal emittance. The full complement of corrector magnets (56 vertical and 54 horizontal dipole correctors and 13 skew quadrupoles), essential for emittance tuning, was preserved. Twelve additional skew quad correctors were implemented in the neighborhood of the arc wigglers to facilitate manipulation of local dispersion and coupling.

We developed and tested optics for operation of the storage ring with beam energies of $1.8 \mathrm{GeV}, 2.0,2.3$, 3.2, 4.0, 5.0 and $5.3 \mathrm{GeV}$ for both electrons and positrons. We achieve our lowest horizontal emittance of $2.6 \mathrm{~nm}$-rad at $2 \mathrm{GeV}$ beam energy with 12 damping wigglers. At $5 \mathrm{GeV}$ the minimum horizontal emittance is $40 \mathrm{~nm}$-rad with 6 damping wigglers powered. All of the conditions are designed to be compatible with on energy injection of multiple bunches so that experimental measurements can be performed efficiently. Figure 4 shows the lattice functions of our $2.0 \mathrm{GeV}$ ultra low emittance optics. The lattice parameters for this optics were previously summarized in Table 2.
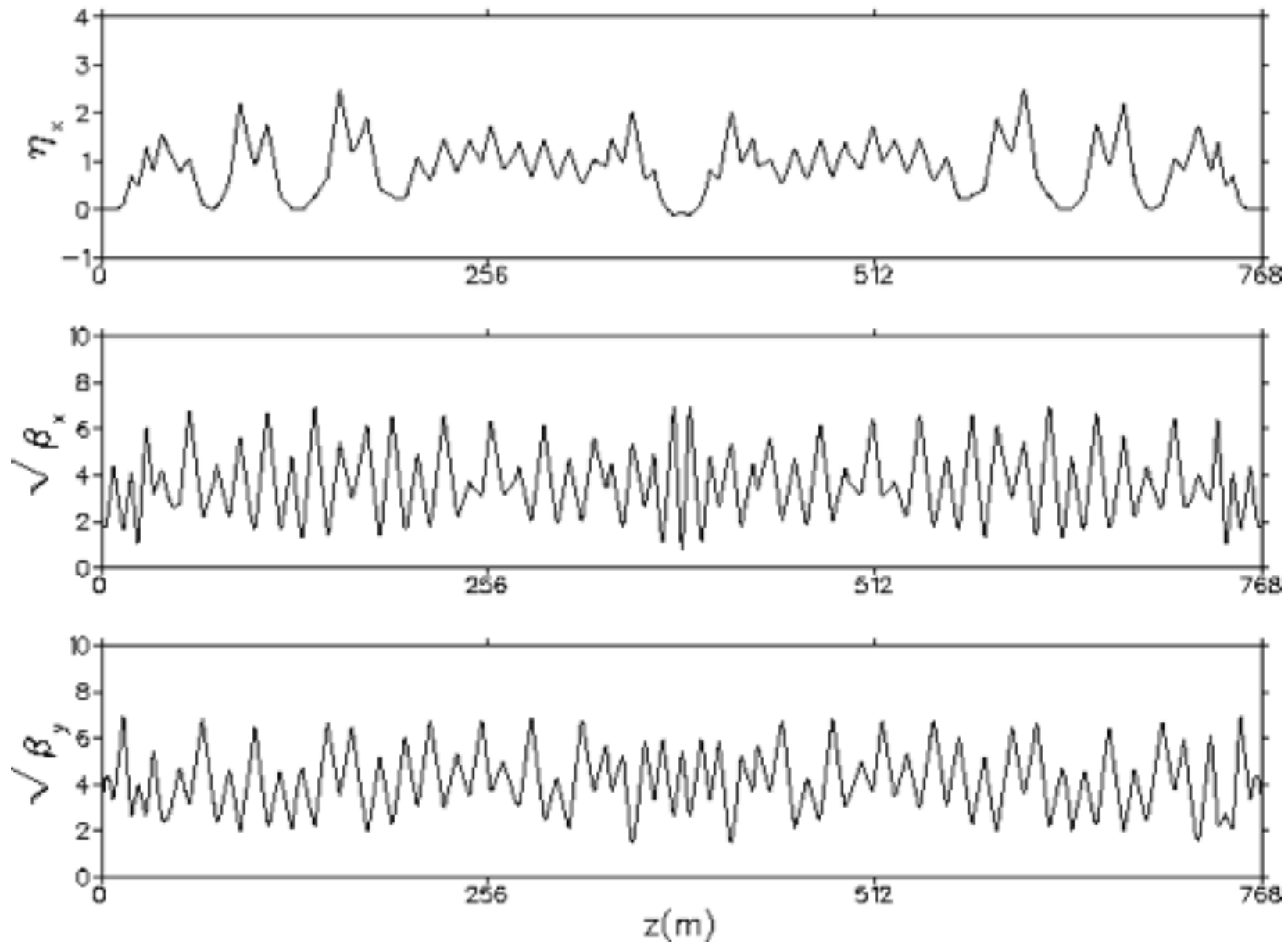

Figure 4: Low emittance lattice. 12 wigglers operating at 1.9T in zero dispersion straights increase radiation damping rate by a factor of 10 and reduce emittance by a factor of 4 . 


\section{international linear collider}

\subsubsection{Machine Modeling}

\subsubsection{Sources of Emittance Dilution}

We consider sources of emittance dilution in the zero current limit. Here we ignore electron cloud interactions, intra beam scattering and other collective effects. In the ideal machine, the closed orbit lies in the horizontal plane. There are no vertical kicks and therefore no vertical dispersion. Vertical emittance arises exclusively from coupling and vertical dispersion generated by magnet misalignments.

It is important to distinguish the normal mode emittance from the horizontal and vertical emittance that we measure in the laboratory. We identify the A-mode with horizontal motion and the B-mode with vertical. Transverse coupling will, in general, locally increase the vertical beam size at the expense of the horizontal. However, it has no effect on the B-mode emittance and the vertical/horizontal aspect ratio can be restored by suitable decoupling. Transverse coupling in regions of finite horizontal dispersion will generate vertical dispersion. The B-mode emittance will increase only if that vertical dispersion is permitted to propagate through a bending magnet or a wiggler.

\subsubsection{Misalignments}

Vertical dispersion can be generated directly by vertical dipole kicks, and indirectly via coupling of horizontal dispersion into the vertical plane. Sources of vertical kicks include quadrupoles vertically offset from the plane of the orbit and horizontal dipole magnets with finite roll. Wigglers produce no net dispersion, but do generate dispersion internally. A wiggler that is rotated (tilted) about the beam axis will generate vertical dispersion internally. We find, however, that the contribution to vertical dispersion from tilted wigglers is negligible in the CESRTA optics.

Tilted quadrupoles couple horizontal dispersion into the vertical plane, as do sextupoles that are vertically displaced from the plane of the ideal orbit. In addition, even perfectly aligned sextupoles introduce coupling if the vertical orbit is distorted by offset quadrupoles or rolled dipoles.

We have performed simulations in order to quantify the sensitivity to alignment errors. The procedure is to create a large number of lattice configurations $(\mathrm{N})$, each with a distribution of misalignments. For each configuration we compute dispersion, coupling and vertical emittance. We find that survey and alignment alone is inadequate to the task of achieving vertical emittance less than 20 pm-rad, and that beam based measurement and correction of residual orbit errors, coupling and vertical dispersion is essential. Therefore we have extended our simulations to include a low emittance tuning procedure. The effectiveness of the low emittance tuning in turn will depend on the precision with which we can measure coupling and dispersion, the availability of corrector magnets, and the quality of our tuning algorithms.

\subsubsection{Machine Model}

Lattice parameters and the effect of misalignments are modeled using the BMAD library [17] routines. The linear guide field elements are characterized by $6 \times 6$ matrices. Sextupoles are modeled using a kickdrift-kick model. The wiggler magnet is modeled as a symplectic map based on an expansion of the field in terms of trigonometric and hyperbolic functions [18]. Misalignments are included by offsetting and/or rotating the relevant phase space coordinates that are tracked through the misaligned element. Once the closed orbit has been computed, magnet matrices are recalculated so that offset and tilted quadrupoles include dipole and skew quad and other feed down components, etc., thus yielding self-consistent Twiss parameters and dispersion function. Finally, the determination of the emittances is based on that set of lattice functions that corresponds to the real closed orbit.

Our simulation [19] is based on a machine model that includes all magnets: quadrupoles, dipoles, damping wigglers, sextupoles, correctors (skew quadrupoles, vertical and horizontal steerings), RF 


\section{international linear collider}

cavities and BPMs. All guide field magnetic elements can be arbitrarily misaligned. BPM absolute and differential measurement resolution and tilts can be specified. The surveyed distributions of alignment errors are shown in Table 3. Misalignments in the simulation are based on those same measured distributions.

Table 3: Surveyed magnet alignment

\begin{tabular}{|c|c|}
\hline Parameter & RMS \\
\hline Quad tilt & $120 \mu \mathrm{m}$ \\
\hline Quad vertical offset & $50 \mu \mathrm{m}$ \\
\hline Dipole roll & $100 \mu \mathrm{rad}$ \\
\hline Sextupole vertical offset & $250 \mu \mathrm{m}$ \\
\hline Wiggler roll & $200 \mu \mathrm{m}$ \\
\hline
\end{tabular}

\subsubsection{BPM Resolution}

There are 100 BPMs in CESR available for beam-based measurements, which are distributed more or less uniformly around the $768 \mathrm{~m}$ circumference. The reproducibility of the measurement of the beam position is established by comparing multiple consecutive measurements to be within $10 \mu \mathrm{m}$. We conclude that the differential position resolution is $10 \mu \mathrm{m}$. The absolute position resolution is based on the reproducibility of the quad centering method [3], and is taken to be $100 \mu \mathrm{m}$.

\subsubsection{Beam Based Measurements for Low Emittance Tuning}

We rely on beam-based measurements to identify sources of vertical emittance dilution including focusing errors, transverse coupling, vertical kicks, and vertical dispersion.

\subsubsection{Phase and Coupling}

Betatron phase and coupling is determined by resonant excitation of the beam at the transverse normal mode tunes (horizontal and vertical or very nearly depending on coupling) and measuring the amplitude and phase of the signal at each of the tunes at all of the beam position monitors [20]. The result is a measure of the beta function and phase advance at each BPM, and 3 of the 4 components of the $2 \times 2$ coupling matrix. The technique has been refined with the implementation of the CESRTA high bandwidth BPM electronics, yielding greater precision and significantly faster turn around time. A single ring-wide measurement and associated analysis can be completed in about 10 seconds. The resolution of the betatron phase measurement is about $1^{\circ}$ corresponding to a beta beat of about $3 \%$. The measurement resolution of the $\overline{C_{12}}$ is about $0.5 \%$. We fit the accelerator model to the measured data using all of the 100 ring quadrupoles and 25 skew quads as variables. The fitted changes to each of those elements are loaded into the magnet power supplies. A measurement of horizontal and vertical betatron phase advance following a round of correction is shown in Figure 5. The corresponding measurement of the $\overline{C_{12}}$ coupling matrix element is shown in Figure 6. An iteration of measurement and correction takes a few minutes. 


\section{international linear collider}
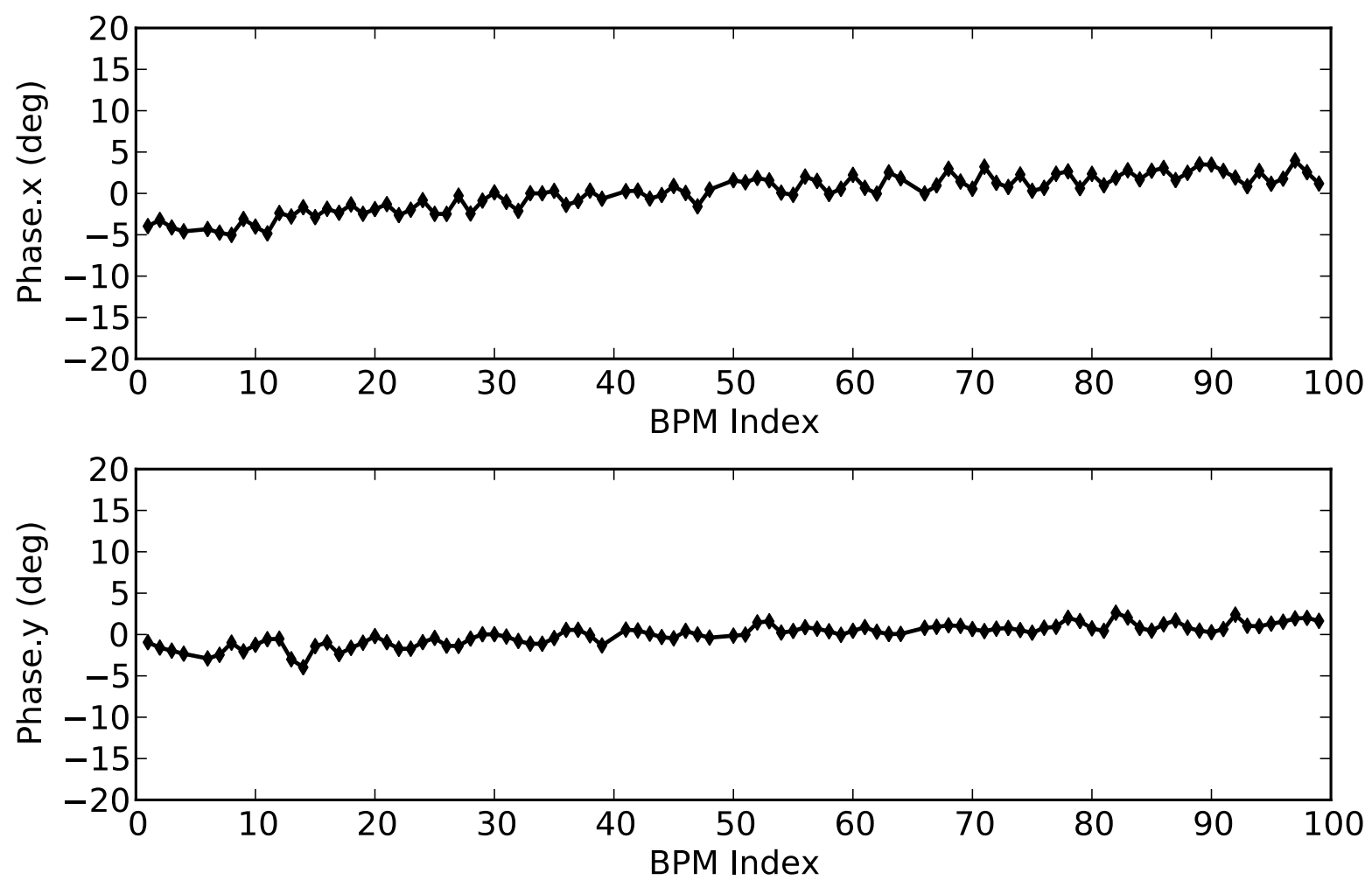

Figure 5: Typical horizontal and vertical betatron phase measurements (measured - design) after corrections. The constant slope indicates a difference in measured and design tunes. Accounting for the tune difference, RMS (measured - design) $\phi_{a}=1.7^{\circ}, \phi_{b}=0.64^{\circ}$. This corresponds to a beta beat of $\sim 3 \%$ and $2 \%$, respectively.

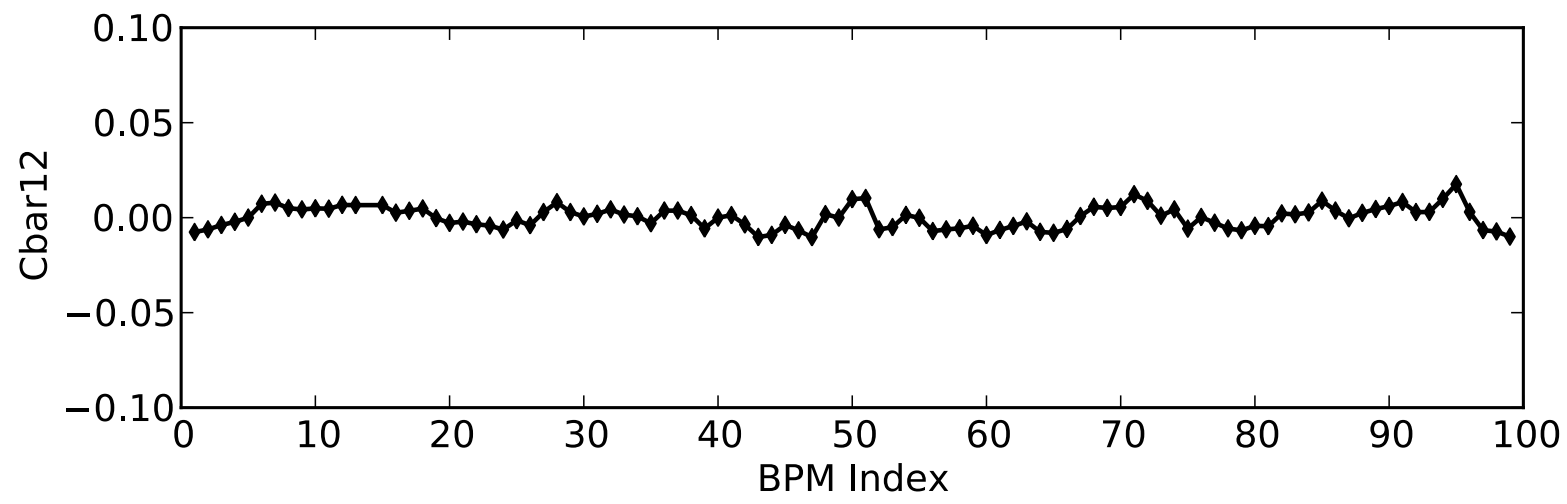

Figure 6: Normalized $\overline{C_{12}}$ coupling after a typical low-emittance correction. The RMS of 0.006 corresponds to an $x-y$ emittance coupling of $0.036 \%$ 


\section{international linear collider}

\subsubsection{Dispersion Measurement}

Vertical dispersion is a principle source of vertical emittance. Our goal is to limit residual vertical dispersion to $<10 \mathrm{~mm}$, which corresponds to a vertical emittance of about $10 \mathrm{pm}-\mathrm{rad}$. We use two distinct techniques to measure dispersion. For the "DC" method we measure the difference of two orbits with different equilibrium energies. The equilibrium energy is changed, by shifting the frequency of the storage ring RF source. The equilibiruim energy can be practically changed by about $0.2 \%$. The BPM resolution for difference measurements (as noted above) is about $10 \mu \mathrm{m}$. Therefore we expect to be able to measure dispersion with resolution of order $\eta \approx(10 \mu \mathrm{m}) / 0.002=5 \mathrm{~mm}$. Indeed we find that the dispersion measurement is reproducible at the level of $5 \mathrm{~mm}$.

Alternatively, we recognize that dispersion is the coupling of the longitudinal motion of the beam into the transverse planes. We can then exploit resonant excitation to measure $z-y$ and z-x coupling just as we do for measuring $x-y$ coupling. We drive the beam at the synchrotron tune and measure the horizontal and vertical motion at that tune at each of the BPMs. The energy amplitude of the driven synchrotron oscillations is approximately the same as the energy difference used for the DC measurement. Therefore we anticipate that the resolution of the two techniques will be comparable. The advantage of this so-called "AC" technique over the DC method is that it is passive, requiring no change to the machine conditions (namely a change in the RF frequency). The resonant excitation measurement can be completed in about 10 seconds. Examples of both AC and DC dispersion measurements are shown in Figure 7.

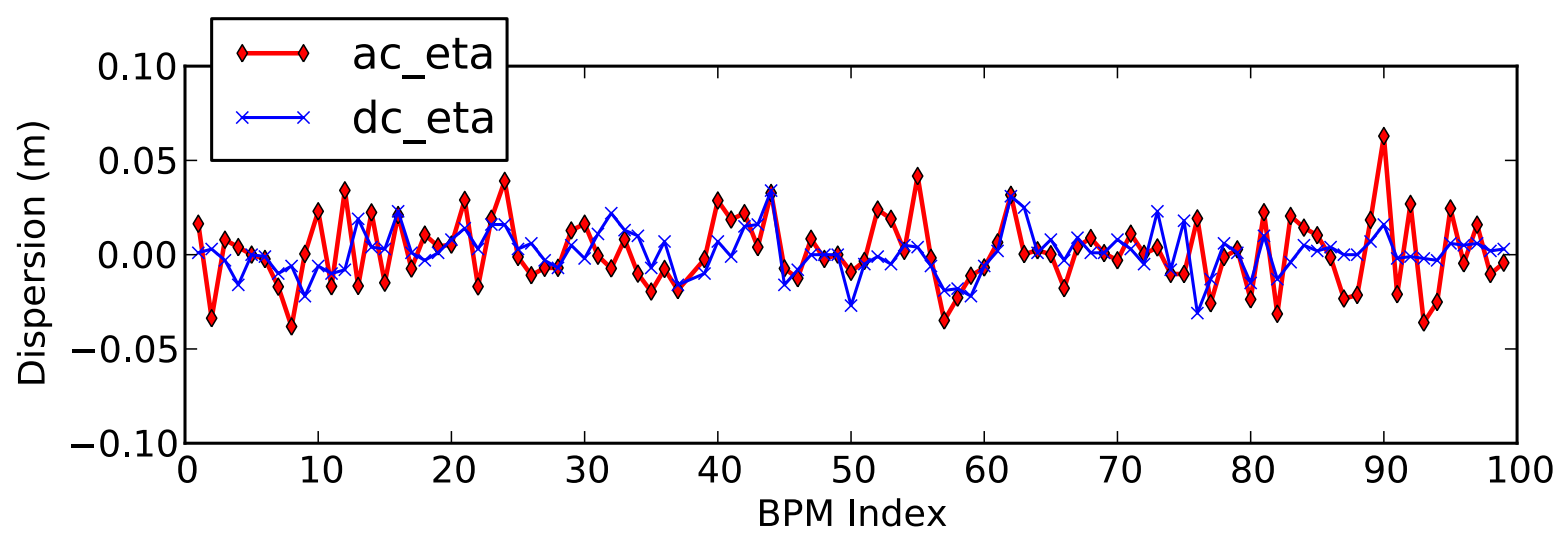

Figure 7: Typical vertical dispersion after a low-emittance correction, measured using both resonant excitation (AC dispersion) and traditional orbit difference (" $D C$ " dispersion) techniques. For AC dispersion, $R M S=19 \mathrm{~mm}$. For traditional dispersion measurement, $R M S=12 \mathrm{~mm}$.

\subsubsection{BPM Systematics}

There are two important systematic limitations of the beam position monitor in the measurement of coupling and vertical dispersion; BPM tilts and BPM button electrode gain variations. If the BPM is physically tilted, then horizontal dispersion can appear in a measurement as vertical. In addition, if the response to the beam of each of the four button electrodes is not identical, there will be an effective BPM coupling. We have developed beam-based techniques to measure button gains at the fraction of a percent level exploiting the turn by turn capability of the new BPM electronics[21]. We then use gain-corrected coupling measurements to determine BPM tilts. 


\section{international linear collider}

\subsubsection{Low Emittance Tuning Procedures}

\subsubsection{Low Emittance Tuning Algorithms}

The low emittance tuning (LET) procedure is based on an iterative series of beam-based measurements and corrections. The procedure is as follows:

1. Measure the orbit and correct using all 55 horizontal and 58 vertical steering correctors (center in the quadrupoles).

2. Measure the betatron phase and transverse coupling by resonant excitation of normal mode tunes. Then, correct the betatron phase to the design phase using all 100 independently powered quadrupoles, and minimize the transverse coupling using 25 skew quads correctors.

3. Re-measure the orbit and transverse coupling, and measure dispersion by resonant excitation of the synchrotron tune.

4. Simultaneously optimize to minimize orbit errors, transverse coupling and vertical dispersion using skew quads and vertical correctors, and load changes to the magnet power supplies.

\subsubsection{Effectiveness of LET Procedure (Simulation)}

We simulate the low emittance tuning procedure by generating guide field configurations with a distribution of misalignments as tabulated above (Table 3) and measurement resolutions described in sections 3.3.3.4-3.3.3.7. The distributions of vertical dispersion and emittance for 200 configurations after correction are shown in Figure 8. We see that typical correction levels in simulation correspond to a $5 \mathrm{~mm}$ residual vertical dispersion and vertical emittance $<5 \mathrm{pm}-\mathrm{rad}$. Insofar as our model accurately represents the real machine misalignments and measurement uncertainty, we anticipate the same level of correction for the storage ring.
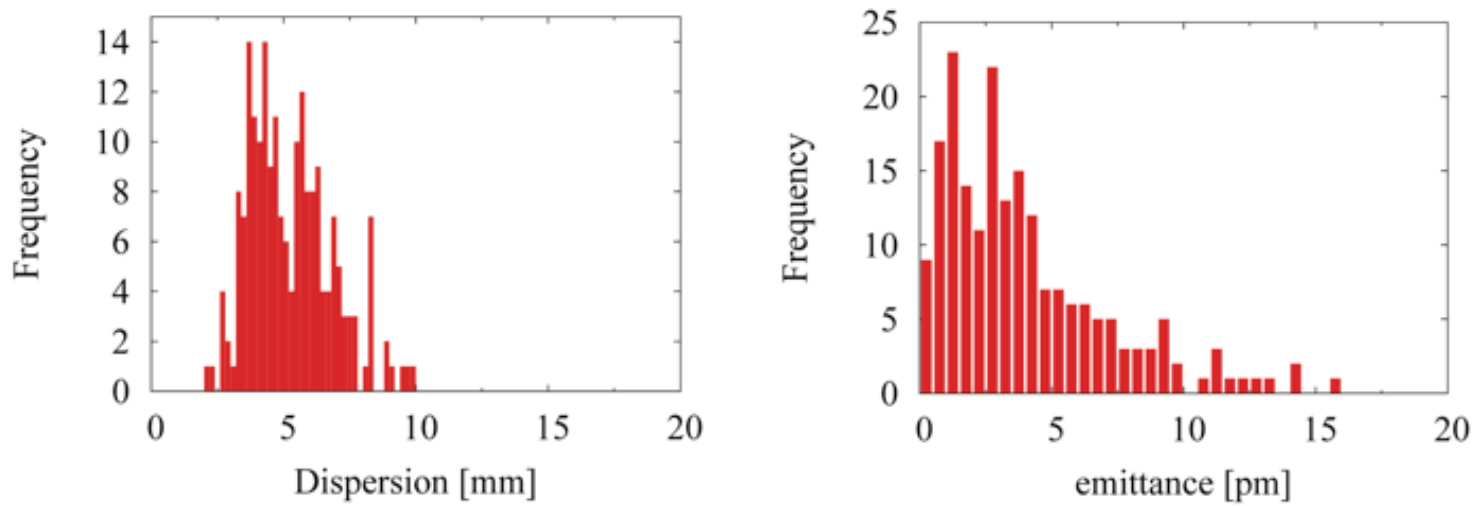

Figure 8: Simulated vertical dispersion and emittance after low emittance tuning procedure for 200 machine configurations with alignment errors and measurement resolution described in the text.

\subsubsection{Experimental Low Emittance Tuning Results}

Lattice errors are minimized using the procedures described above. The effectiveness of the correction of the optics at the conclusion of the LET procedure is summarized in Table 4, for the December 2010 CESRTA machine studies. The measured vertical dispersion of $14 \mathrm{~mm}$ is beyond the tail of the simulated distribution (Figure 8a). We measure the vertical beam size with the xBSM. The measured vertical emittance of 6 pm-rad falls well within the simulated distribution (Figure 8b). We suspect that the discrepancy between measured and modeled dispersion is some systematic effect of the AC dispersion technique that has yet to be understood.

The beam size is based on an average of 100 single turn measurements. Figure 1 shows the x-ray detector and a fit to a single turn measurement. The beamsize monitor updates every few seconds, allowing the possibility of empirical tuning of vertical emittance. Empirical adjustment of betatron tunes, and of closed 


\section{international linear collider}

coupling and vertical dispersion bumps, are found to reduce vertical beam size by a few microns below that resulting directly from the LET procedure. Iteration of the LET procedure alone consistently yields $<10$ pm-rad vertical emittance.

Table 4: Correction levels after iterating LET correction procedure and empirical tuning.

\begin{tabular}{|c|c|}
\hline Parameter & RMS Value \\
\hline RMS Betatron Phase Error & $1.2^{\circ}$ \\
\hline RMS Beta Beat & $0.22 \%$ \\
\hline RMS Betatron Coupling $\left(\overline{C_{12}}\right)$ & 0.006 \\
\hline RMS Vertical Dispersion & $14 \mathrm{~mm}$ \\
\hline Vertical Emittance & $6.0 \mathrm{pm}-\mathrm{rad}$ \\
\hline
\end{tabular}

\subsection{The Electron Cloud R\&D Program at CESRTA}

\subsubsection{Electron Cloud Build-Up and Mitigation Studies}

RFAs deployed at approximately 30 locations around CESR have enabled the detailed study of local cloud build-up in a variety of vacuum chambers under a range of experimental conditions [22,23]. The RFAs provide a time-averaged current readout at each location. The majority of deployed RFAs utilize a segmented design to provide geometric information about the EC build-up around the azimuth of the vacuum chamber. RFA data taken in vacuum chambers fabricated with EC mitigations provides the foundation for comparison of the efficacy of different EC mitigation methods. An effort is underway to model this RFA data (see Sect. 3.4.3) in order to determine the secondary electron yield (SEY) and photoelectron yield (PEY) parameters of the vacuum chambers treated with mitigations [23-25]. The vacuum chambers with EC mitigations come from various sources. Several chambers have been prepared by the Cornell group for these studies. In addition, a significant number of chambers and mitigation treatments to chambers have been provided by our collaborators. In particular, wiggler vacuum chambers with a range of mitigations applied have been produced in collaboration with our KEK, LBNL, and SLAC colleagues. After the conclusion of the BaBar/PEP-II B-physics program, the EC hardware that had been deployed in the positron low energy ring (LER) of PEP-II [26,27] was transferred to CESR to continue the studies that had not yet been completed at PEP-II. Finally, vacuum chambers with various low SEY coatings have been prepared in collaboration with our colleagues at CERN, KEK, LBNL and SLAC.

In addition to the RFA studies, TE Wave transmission methods [28] are being used to characterize the build-up around the ring and a simulation effort is underway to take full advantage of these results [2931]. A final method to study local EC build-up is shielded pickup measurements [32], which provide additional constraints on the vacuum chamber surface parameters for the chambers in which they're installed. Table 5 summarizes the range of chamber surfaces and mitigation methods that were prepared for testing during Phase I of the CESRTA R\&D program.

Figure 9 shows a comparison of the performance of various chamber surfaces in a dipole field along with a plot of the evolution of the transverse distribution of the EC that develops in the dipole chamber as a function of beam current. While coating with a low SEY material such as titanium nitride (TiN) significantly reduces the growth of the EC in this environment, the use of a grooved surface with TiN coating is clearly superior. 


\section{international linear collider}

Table 5: Vacuum chambers fabricated for testing during Phase I of the CESRTA R\&D program. Checks indicate chambers for which data has already been acquired.

\begin{tabular}{|c|c|c|c|c|c|}
\hline Mitigation & Drift & Quadrupole & Dipole & Wiggler & $\begin{array}{c}\text { Institutions Providing } \\
\text { Chambers }\end{array}$ \\
\hline Al & $\checkmark$ & $\checkmark$ & $\checkmark$ & & CU, SLAC \\
\hline $\mathrm{Cu}$ & $\checkmark$ & & & $\checkmark$ & $\begin{array}{l}\text { CU, KEK, LBNL, } \\
\text { SLAC }\end{array}$ \\
\hline TiN on Al & $\checkmark$ & $\checkmark$ & $\checkmark$ & & CU, SLAC \\
\hline TiN on $\mathrm{Cu}$ & $\boldsymbol{V}$ & & & $\checkmark$ & $\begin{array}{c}\text { CU, KEK, LBNL, } \\
\text { SLAC }\end{array}$ \\
\hline Amorphous C on Al & $\checkmark$ & & & & CERN, CU \\
\hline Diamond-like C on Al & $\checkmark$ & & & & CU, KEK \\
\hline NEG on SS & $\checkmark$ & & & & $\mathrm{CU}$ \\
\hline Solenoid Windings & $\checkmark$ & & & & $\mathrm{CU}$ \\
\hline Fins w/TiN on Al & $\checkmark$ & & & & SLAC \\
\hline $\begin{array}{c}\text { Triangular Grooves on } \\
\mathrm{Cu}\end{array}$ & & & & $\boldsymbol{V}$ & $\begin{array}{c}\text { CU, KEK, LBNL, } \\
\text { SLAC }\end{array}$ \\
\hline $\begin{array}{c}\text { Triangular Grooves } \\
\text { w/TiN on Al }\end{array}$ & & & $\boldsymbol{V}$ & & CU, SLAC \\
\hline $\begin{array}{c}\text { Triangular Grooves } \\
\text { w/TiN on } \mathbf{C u} \\
\end{array}$ & & & & $\boldsymbol{V}$ & $\begin{array}{c}\text { CU, KEK, LBNL, } \\
\text { SLAC }\end{array}$ \\
\hline Clearing Electrode & & & & $\checkmark$ & $\begin{array}{c}\text { CU, KEK, LBNL, } \\
\text { SLAC }\end{array}$ \\
\hline
\end{tabular}

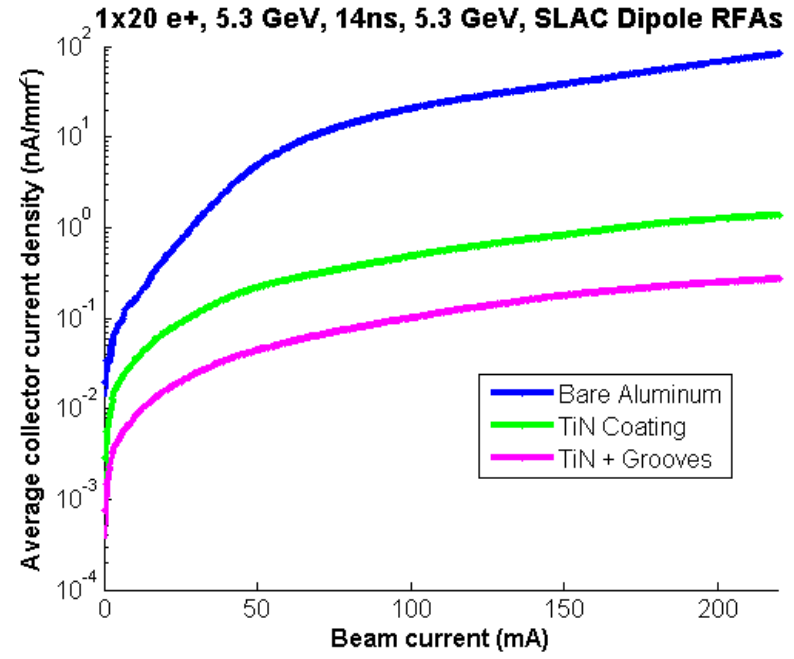

Run \#1912 (1x20 e+, 5.3 GeV, 14ns): SLAC RFA 4 (Al) Col Curs

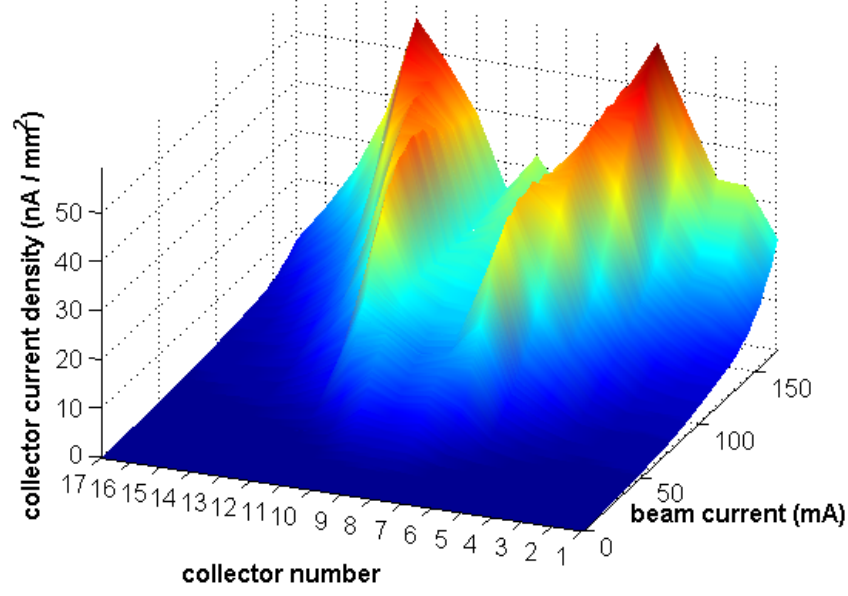

Figure 9: Plot (left) showing the measured RFA current in a dipole versus beam current with a 20 bunch positron train for a bare Al surface, TiN-coated surface and a grooved surface with TiN-coating. The efficacy of the grooved surface for suppressing the EC is evident. The second plot (right) shows the transverse shape of the EC signal in the dipole RFA (Al chamber surface) as a function of beam current. (Collector 9 is at the center of the top of the vacuum chamber). 


\section{international linear collider}

Figure 10 shows two of the mitigation methods that have been tested in the CESRTA high field damping wigglers: triangular grooves and a clearing electrode. The clearing electrode is a very thin structure developed at KEK [33-35], which offers very good thermal contact with the vacuum chamber and minimal impact on the chamber aperture. A bare $\mathrm{Cu}$ surface and a TiN-coated $\mathrm{Cu}$ surface have also been tested. The left plot in Figure 11 shows a comparison of the EC growth as a function of beam current with each of these surfaces. Our observations indicate that the best cloud suppression in the wiggler region is obtained with the clearing electrode. Similar electrodes have also been successfully tested in the positron ring of the KEKB B-factory and are now being incorporated into the SuperKEKB positron ring design. This style of electrode offers a relatively low impedance and very good thermal stabilization to the vacuum chamber.

One additional wiggler comparison remains, the testing of a grooved surface with TiN coating. This test chamber has recently been installed in CESR and complete testing will be carried out during Phase II of the R\&D program. The right plot in Figure 11 shows the transverse distribution of the EC present in the vertical field region of the wiggler ( $\mathrm{Cu}$ surface) as a function of RFA retarding grid voltage, which probes the energy spectrum of the EC.

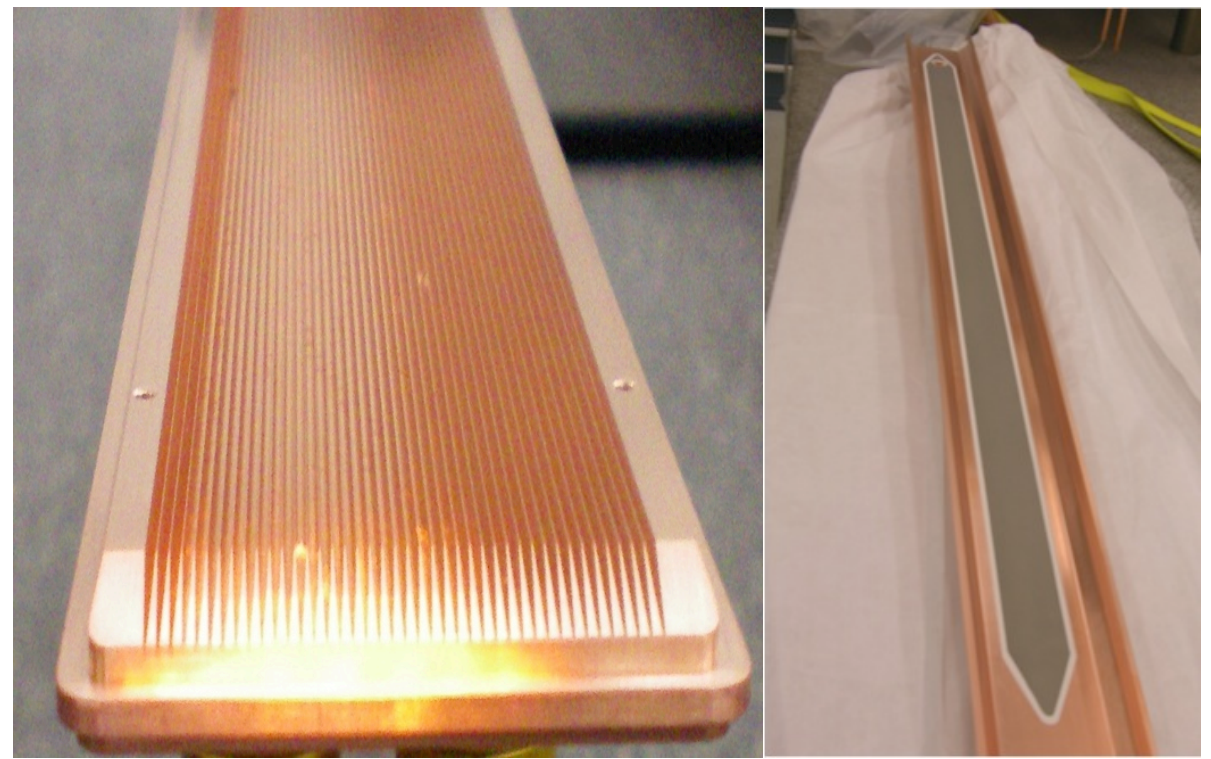

Figure 10: The left photo shows a grooved Cu insert with $21.8^{\circ}$ triangular grooves having $1 \mathrm{~mm}$ pitch for testing in a CESRTA wiggler while the right photo shows a thin clearing electrode applied with a thermal spray method to the bottom half of another CESRTA experimental wiggler chamber. 


\section{international linear collider}

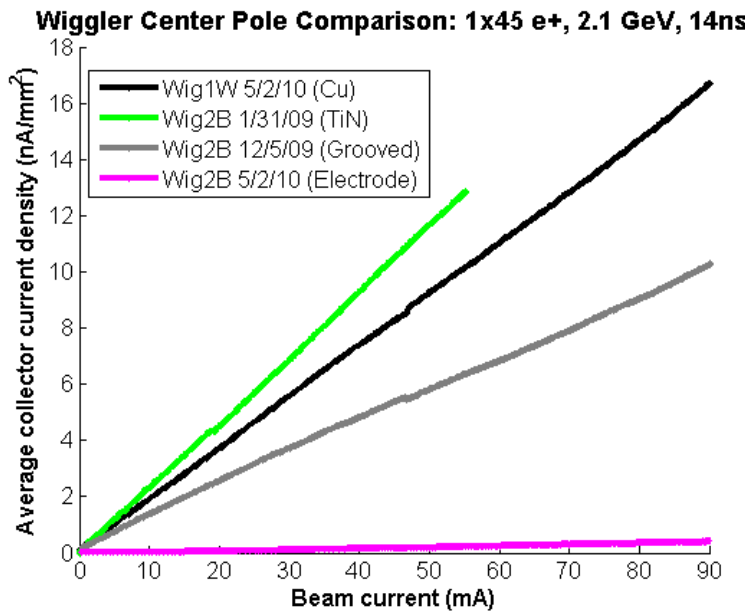

Run \#2955 (1x45x.75mA e+, $2.1 \mathrm{GeV}, 14 n s):$ Wig1W Center Pole

Figure 11: Plot (left) showing the measured RFA current in a wiggler versus beam current with a 20 bunch positron train for a bare Cu surface, TiN-coated Cu surface, and a grooved Cu surface, and a clearing electrode. The efficacy of the clearing electrode for suppressing the EC is clearly evident. The second plot (right) shows the transverse shape of the EC signal in the wiggler RFA as a function of retarding voltage.

Studies of the EC build-up in drift and quadrupole regions have also yielded important results. Drift measurements have been used to compare the performance of various coatings. A new coating of some interest is the amorphous carbon coating developed at CERN [36] for use in the Super Proton Synchrotron (SPS). Tests at CESRTA have afforded the opportunity to study the performance of this coating in the presence of synchrotron radiation. Initial studies show that the EC mitigation performance of amorphous carbon is comparable to that of TiN and that its vacuum performance is reasonable in an environment with significant photon flux. Continued testing will provide information about the long term durability of this very promising coating. Vacuum chambers in quadrupole magnetic fields can show significant EC build-up. Concerns about long-term trapping of the EC in quadrupole fields [37] suggest that EC mitigation be incorporated into the ILC DR quadrupole vacuum chambers. Tests in CESRTA have demonstrated the effectiveness of TiN coating in this quadrupole field region.

\subsubsection{Studies of EC-induced Beam Dynamics with Low Emittance Beams}

The CESRTA low emittance tuning (LET) effort provides the basis for studying the emittance-diluting effects of the EC in a regime approaching that of the ILC damping rings. As of early 2010, the LET program had resulted in reliable operation at or below the Phase I emittance target of $\varepsilon_{\mathrm{y}} \leq 20 \mathrm{pm}-\mathrm{rad}$ [38] for both single- and multi-bunch operation as confirmed by xBSM measurements of the vertical beam size [39]. As of the conclusion of 2010, vertical emittances of $\varepsilon_{\mathrm{y}}<10 \mathrm{pm}-\mathrm{rad}$ had been achieved.

A number of beam dynamics studies have been conducted in order to fully characterize the impact of the EC on beams in CESR. As the EC builds up along a bunch train, the focusing effect of the cloud on the beam causes the natural frequency of oscillation of each bunch (ie, the horizontal and vertical betatron tunes) to shift with respect to the preceding bunch. Measurements of this EC-induced coherent tune shift $[40,41]$ for trains of electron and positron bunches, as well as for witness bunches at various positions behind a leading train, have provided an important probe of the integrated effect of the cloud around the ring. Systematic measurements over a wide range of beam conditions (varying beam energy, emittance, bunch current, bunch spacing and train lengths) are being used to validate more thoroughly our EC models and have led to improved simulations, eg, for the ring photon propagation model [42], which are now being applied to the ILC DR. 


\section{international linear collider}

A principal deliverable of the CESRTA program is the characterization of instability thresholds and emittance-diluting effects in the ultra low vertical emittance regime [43-45]. Figure 12 shows the observed beam motion spectrum for each bunch along a train obtained in these conditions. As described in the preceding paragraph, the development of the horizontal and vertical tune lines, denoted by $\mathrm{F}_{\mathrm{h}}$ and $\mathrm{F}_{\mathrm{v}}$, along the bunch train provides information about the $\mathrm{EC}$ density experienced by each bunch. For a positron train, the attractive force of the bunch on the cloud pinches the cloud into the bunch and can lead to the development of an oscillation of the tail with respect to the head. This head-tail instability is expected to induce characteristic sidebands in the bunch motion spectrum. In the plot in Figure 12 the onset of the spectral lines denoted by $\mathrm{F}_{\mathrm{v}} \pm \mathrm{F}_{\mathrm{s}}$ part way along the bunch train indicates where the EC density build-up has become sufficient for the onset of the instability. A second observable associated with this instability is a growth in the vertical beam size as measured along the train. Figure 13 shows bunch-bybunch beam size development along bunch trains with 3 different intensities. As the bunch currents are increased, the bunch number in the train at which beam size blow-up occurs moves earlier in the train due to the more rapid build-up of the EC. By studying both the spectral and beam size information as a function of various parameters (eg, bunch intensity, vertical emittance, bunch spacing, chromaticity, feedback conditions, and beam energy) and comparing with simulation [46,47], we will be able to validate the simulations in a regime approaching that of the ILC DR and ensure that our projections of the expected positron DR performance are accurate.

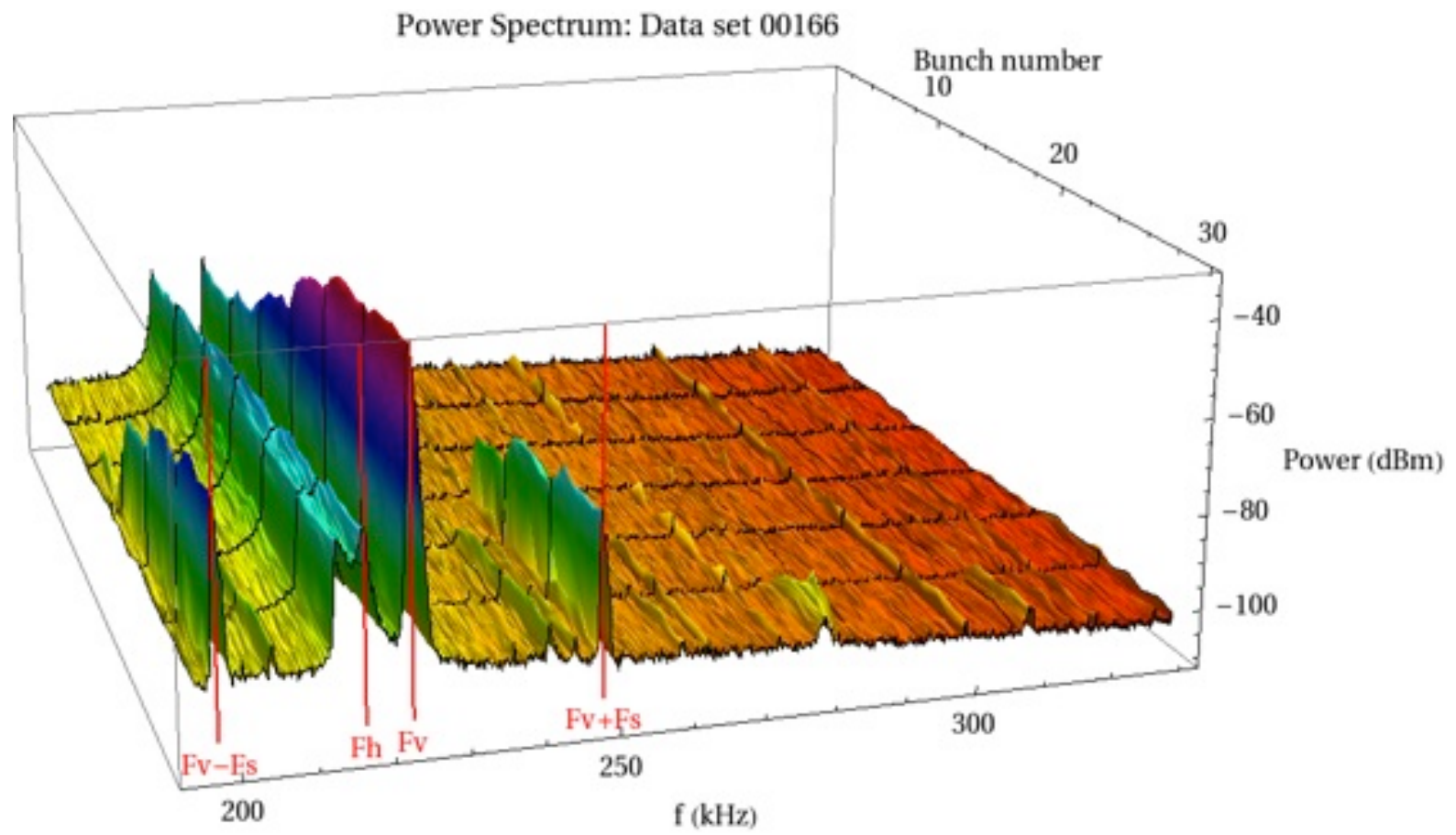

Figure 12: Bunch-by-bunch power spectrum for a positron train with a nominal bunch current of $0.75 \mathrm{~mA} /$ bunch. The horizontal $\left(F_{h}\right)$ and vertical $\left(F_{v}\right)$ tunes are clearly visible for all bunches. The onset of the sidebands labeled as $F_{v} \pm F_{s}$ are consistent with the onset of a head-tail instability around bunch number 15 in the train. 


\section{international linear collider}

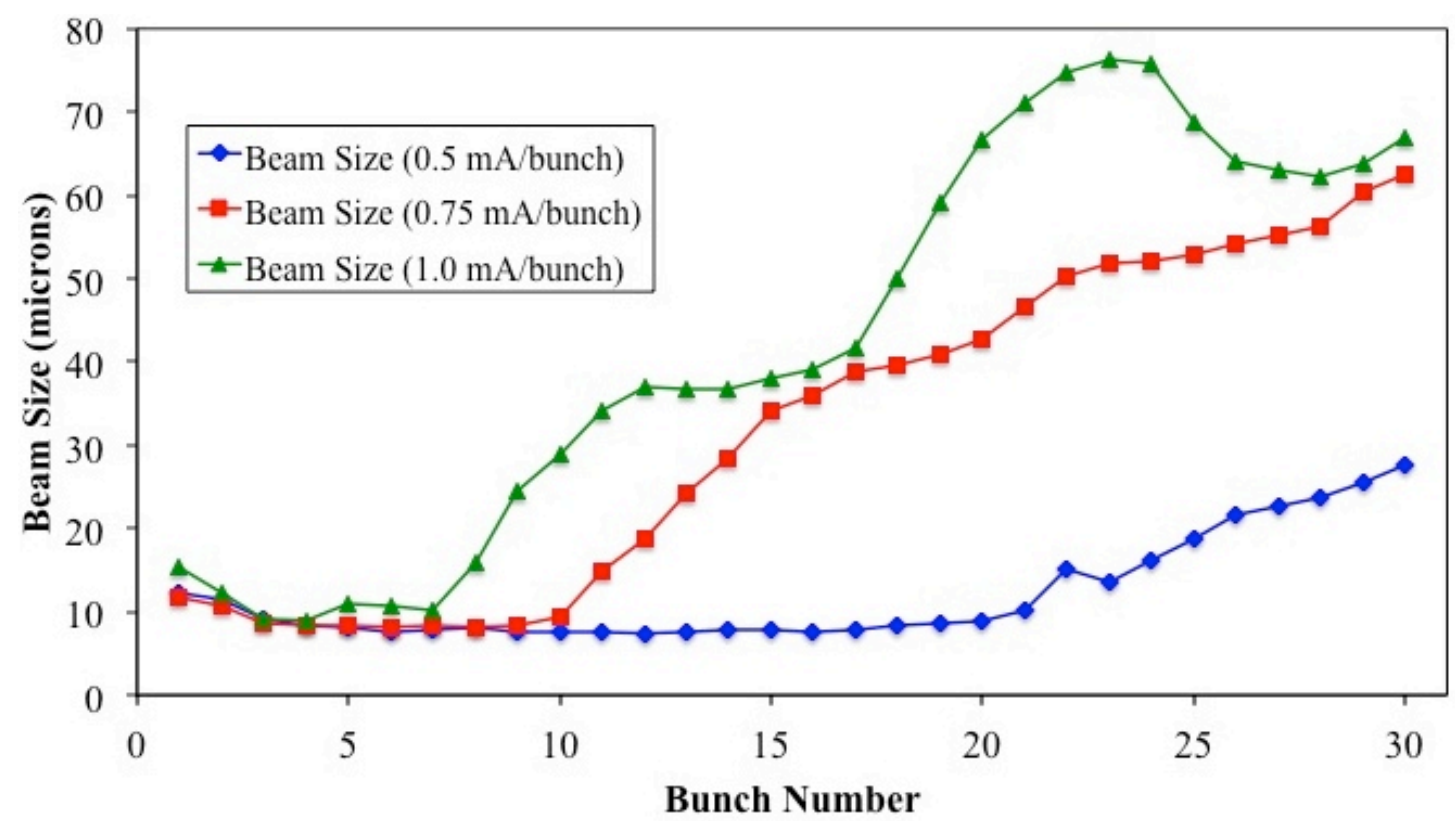

Figure 13: Bunch-by-bunch beam sizes based on turn-by-turn fits for each bunch for 30 bunch trains of varying current $\left(0.8,1.2\right.$, and $1.6 \times 10^{10}$ particles/bunch). As the bunch currents are increased, the point in the train at which the EC density is high enough to cause emittance and beam size growth moves to earlier points in the train.

\subsubsection{Modeling Codes - Projections for the Damping Ring Performance}

We have developed a number of modeling codes; to help to interpret the electron cloud measurements, to guide the experimental program, and to form the basis for incorporating our results into the design of the ILC damping ring.

\subsubsection{Electron Cloud Simulation Codes}

A broad-based program of developing, comparing and benchmarking electron cloud build-up simulation codes was initiated in the CESRTA collaboration in 2008 and continues today. We have used CESRTA parameters as input to the simulation codes ECLOUD [48], CLOUDLAND [49], POSINST [50], WARPPOSINST [51] and PEHTS [52], and compared the results against measurements. By iterating this process, we are able to pin down parameters that are not well known and thereby make more reliable extrapolations to the future ILC damping rings. The main (indeed, the only) parameters that are not well known are those pertaining to the electronic surface properties, ie. photon reflectivity, photoemission and secondary electron emission.

Essential components of the codes are photoelectron generation models, time-sliced calculations of macroparticle dynamics including beam kicks, the space charge force from the cloud itself, and the Lorentz forces of ambient magnetic fields, and the model describing the various components of the secondary yield (SEY). The ECLOUD and POSINST models use two-dimensional approximations for the electric fields and have successfully reproduced a variety of coherent tune shift measurements, as well as local RFA and shielded-pickup measurements. These 2D codes are relatively fast, and are generally successful in predicting the features of the electron cloud in regions of the ring that are essentially 2D, namely field-free regions and long magnets, especially dipoles. The CLOUDLAND and WARP/POSINST codes are 3D, hence far more computationally expensive, and have been used to model the electron cloud in essentially $3 \mathrm{D}$ regions such as quadrupole and wiggler magnets, thus providing information on the long-term trapping of electrons. In addition, it should be pointed out that ECLOUD, POSINST and CLOUDLAND are build-up codes, assuming the beam is a prescribed function of space and time and therefore does not respond to the electron cloud. WARP-POSINST, in addition to being 3D, 


\section{international linear collider}

incorporates the dynamics of the beam so that the beam and the electron cloud respond to their mutual simultaneous influence.

Because of differences in the various cloud modeling codes, especially in the handling of secondary electron yield (SEY), one can often get inconsistent predictions for the same apparent set of parameters. To help sort out these differences, a program of code benchmarking was undertaken at Cornell, where here "benchmarking" refers specifically to comparisons of the simulation programs with each other, as opposed to comparisons with data. The goals of this effort were twofold: first, to investigate the source of any disagreements between the codes; and second, to verify that the codes come into agreement when these differences are removed. The primary conclusion of this benchmarking effort was that discrepancies in the code results arise from specific differences in the primary and secondary emission models, and that the results can be brought into agreement once these differences are removed [53].

The electron cloud induced coherent tune shift provides a striking example of the sensitivity of the simulations to the model parameters. Consider a train of 20 bunches. The EC formation is initiated by the $\mathrm{x}$-ray photons emitted by the bunches in the train striking the walls of the vacuum chamber. The density of the cloud increases along the train, with the lead bunch witnessing a relatively low density cloud and the tail bunch the highest density. The EC focuses the bunches in the positron beam, causing their tunes to shift. Indeed the tune shift is a measure of the density of the cloud encountered by each bunch. Figure 14 shows the tune versus bunch number for a train of positrons followed by a witness bunch placed at some delay behind the train. The growth and evolution of the cloud for the simulation results is modeled with POSINST. The tune shift increases along the train as anticipated. The points at bunch numbers greater than 20 correspond to witness bunches that are placed at successive time intervals beyond the end of the train. The falling tune shifts of the various witness bunches mark the decay of the cloud. The black points in both plots are data. The circles correspond to horizontal tune shift and the squares to vertical. The red, blue and green points in the left plot are simulation results based on 3 different values of the secondary emission yield (SEY). The best fit on the left plot is for SEY=2. The photon distribution used to generate the primary electrons in the left plot is based on direct radiation, with $15 \%$ reflectivity, and a Gaussian photoelectron spectrum. The data in the left and right plots is the same, but the simulation on the right incorporates the Synrad3D photon distribution (see below) as well as SEY=2, the best fit SEY in the left plot. Note that the horizontal tune shift data is in significantly better agreement on the right than the left. We find that the tune shift measurement is remarkably sensitive to details of the photon distribution and scattering model.

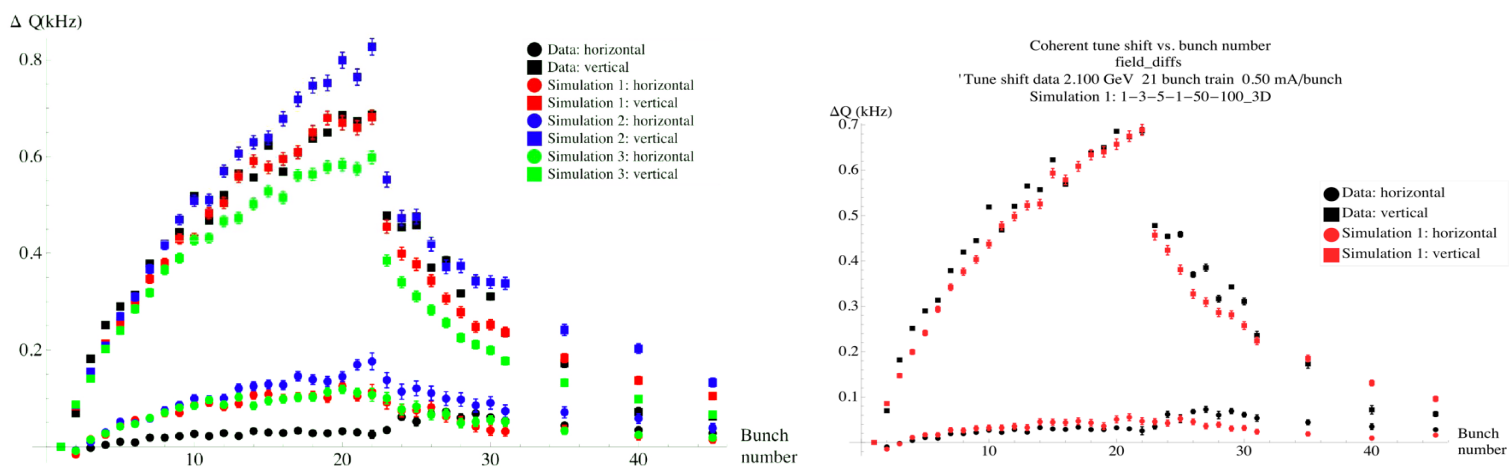

Figure 14: Tune shifts vs. bunch number, for $2.1 \mathrm{GeV}$ data set. The black points are data. Circles are horizontal tune shift and circles are vertical. The blue, red and green points (left plot) are computed (using POSINST) from SYNRAD[2D] for $S E Y=1.8,2.0,2.2$ respectively. The red points (right plot) are computed(using POSINST) from SYNRAD $3 D$ photon simulations. 


\section{international linear collider}

Another probe of the dependence of cloud evolution on model parameters is provided by shielded pickups. While the RFAs provide time-averaged information about the cloud density function, the shielded pickup data resolves the time dependence as illustrated in Figures 15 and 16. The witness bunch experimental method consists of generating a cloud with a leading bunch, then accelerating cloud electrons into the shielded pickup detector with a trailing bunch at various delay times. The magnitude and time structure of the signal from the leading bunch is determined by the reflective properties of the vacuum chamber and by the energy-dependent quantum efficiency. Figure 15 shows the results of three sets of simulations (using ECLOUD) with various values for the elastic yield parameter $\delta_{0}$. The data is from measurements with a shielded pickup in a bare aluminum chamber. Figure 16 shows similar measurements from a pickup in a titanium nitride coated aluminum chamber. We find that the optimum value for the elastic yield is very different in the bare aluminum and TiN chambers.
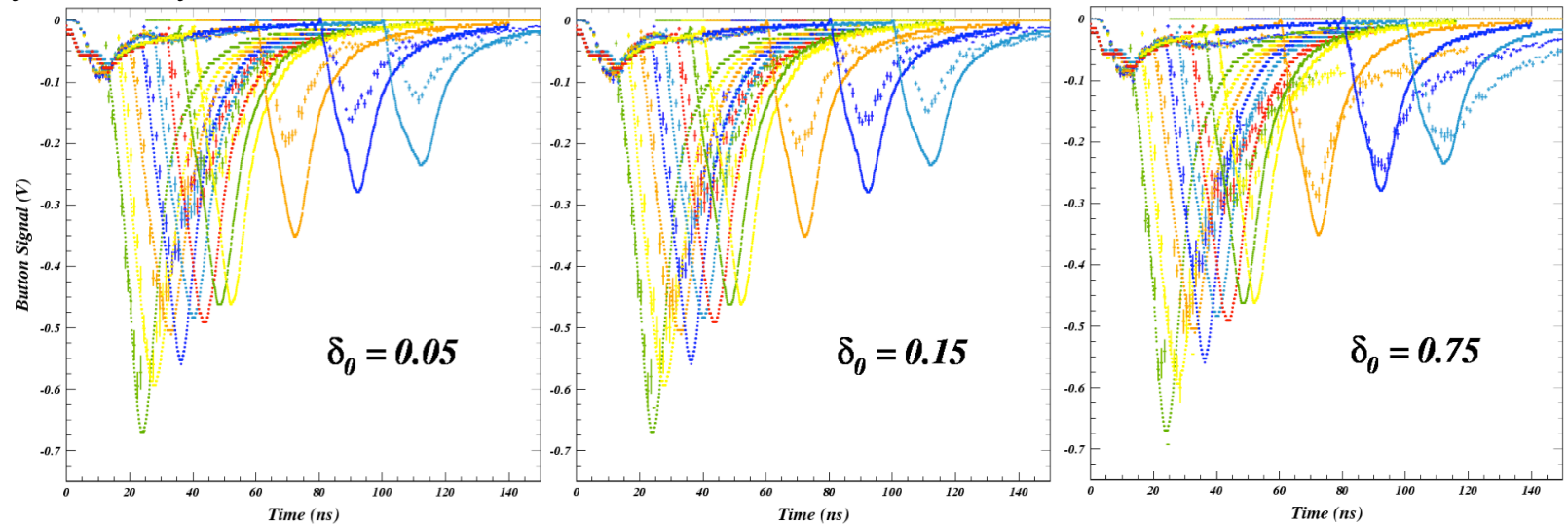

Figure 15: Witness bunch study with the uncoated aluminum chamber. The smooth curves are the shielded pickup signals, the different colors corresponding to different delay of witness with respect to initial bunch.. Eleven two-bunch scope traces are superposed in each of the three plots, whereby the delay of the witness bunch ranges from 12 to $100 \mathrm{~ns}$. The modeled signals are shown with the statistical error bars corresponding to the number of macroparticles contributing to the signal. The magnitudes of the modeled signals at large witness bunch delay clearly show the dependence on the elastic yield parameter $\delta_{0}$ as it is varied from 0.05 to 0.75 . The most consistent description of the measured signals is given by a value of $\delta_{0}=0.75$.
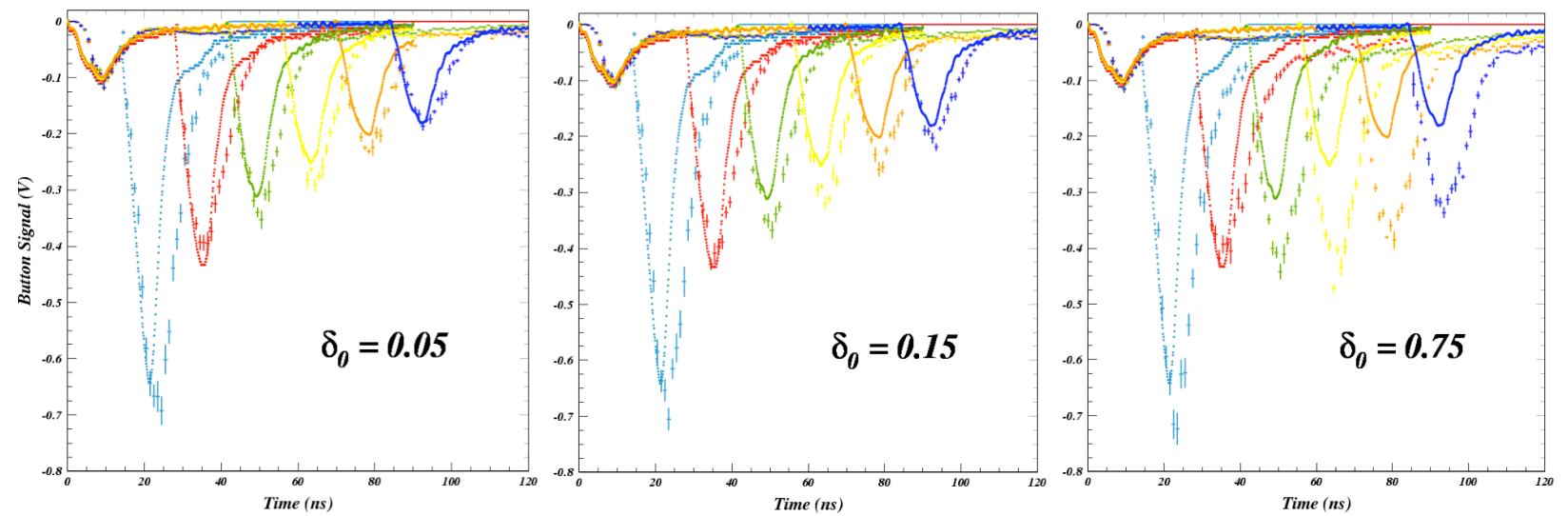

Figure 16: Witness bunch study with the titanium-nitride-coated aluminum chamber. The smooth curves are the digitized shield pickup signals, each color corresponding to a different delay of the witness bunch with respect to initial bunch. Six two-bunch scope traces are superposed in each of the three plots, whereby the delay of the witness bunch ranges from 14 to $84 \mathrm{~ns}$ in 14ns increments. The magnitudes of the modeled signals at large witness bunch delay, clearly show the dependence on the elastic yield parameter $\delta_{0}$ as it is varied from 0.05 to 0.75 . The most consistent description of the measured signals is given by a value of $\delta_{0}=0.05$. 


\section{international linear collider}

\subsubsection{SYNRAD3D}

The SYNRAD3D program is based on the BMAD[17] library that is used for modeling relativistic charged particle beams. SYNRAD3D includes scattering from the vacuum chamber walls using X-ray data from an LBNL database [54]. Photons are generated with respect to the particle beam's closed orbit, so the effect of variations in the orbit can be studied. Simulated photons are tracked until they hit the wall, where the probability of being scattered is determined by their energy and angle of incidence. The vacuum chamber wall is characterized at a number of longitudinal positions by its cross-section.

As an example of the complexity of the photon distribution, we consider the CESR ring with a $5.3 \mathrm{GeV}$ positron beam, and use SYNRAD3D to simulate photon emission only in the arc dipole at B12W. The vacuum chamber is a simple ellipse. The photons are generated only in the upstream end of this dipole but propagate downstream and can scatter. Photons generated by the beam in B12W strike the B12W vacuum chamber a short distance downstream. Some are absorbed here, but most scatter and strike the vacuum chamber further downstream, in $\mathrm{B} 13 \mathrm{~W}$ where more are absorbed, but many others scatter again. Figure 17 shows the photon trajectories in three dimensions. Photons from the source (on the right) propagate and strike the vacuum chamber. Blue dots represent absorption sites. For this simple example, in which the photon source is localized longitudinally, the absorption site locations tend to be clumped in several clusters (at the location of downstream dipoles), with decreasing intensity further from the source.

15

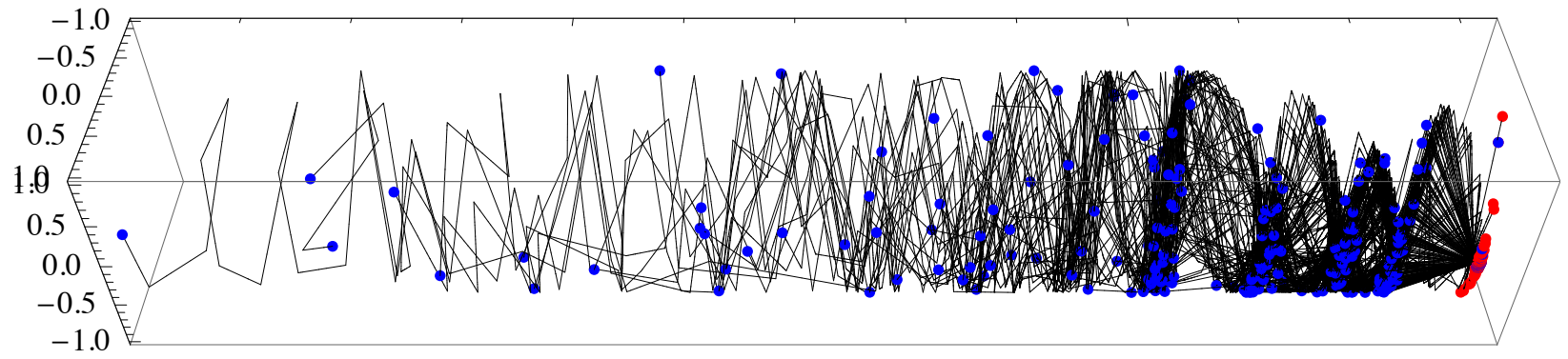

Figure 17: Photon trajectories from the CESR B12W dipole in three dimensions. The photon source is on the right. Black lines are trajectories, and blue dots are photon absorption sites. The transverse geometry has been distorted from an ellipse to a circle, and the longitudinal dimension has been rectified and divided by 10, for purposes of illustration.

\subsubsection{CMAD}

CMAD [55,56] is a many-particle systems program to simulate the transport of charge particle beams in an accelerator lattice in the presence of electron cloud interaction. The program tracks the beam across the full lattice of the accelerator while computing the beam-electron cloud interactions within every element of the lattice. The interaction between the beam and electron cloud is modelled using the particle-in-cell (PIC) algorithm. Currently we have performed simulations for 512 turns using CESRTA parameters. CESRTA has a synchrotron tune of 0.055 . This translates to about 25 synchrotron periods, which is sufficient for studying head-tail oscillations but not so for studying slow emittance growth at low cloud densities. While the task of performing longer simulations is currently in progress, we have been able to get several interesting results with 512 turns that have shown behaviors observed at CESRTA.

Consider the results of a study made with varying cloud densities. In machine measurements we typically use 30 to 45 bunches in a train. Depending upon its properties, each bunch creates a certain amount of cloud and as a result the trailing bunches experience a higher cloud density compared to the leading ones. CESRTA instrumentation has the capability to measure the turn-by-turn position and the beam size of each bunch in a train. CMAD tracks a single bunch so, in order to simulate the effect of different bunches along the train, we need to perform a set of independent calculations using pre-specified cloud densities corresponding to the build-up along the train.. The cloud densities seen by different bunches can be estimated from build-up codes (like POSINST and ECLOUD). At present CMAD assumes a uniform 


\section{international linear collider}

initial distribution of electrons. Work is underway to generalize the program be able to use any distribution as an initial condition.

Figure 18 shows the spectra of the centroid motion of bunches encountering various cloud densities. We see that the betatron tune is gradually shifted with increasing cloud densities. The synchrotron sidebands are evident, indicative of head-tail motion. The CMAD results are in qualitative agreement with measurements, an example of which is shown in Figure 12.
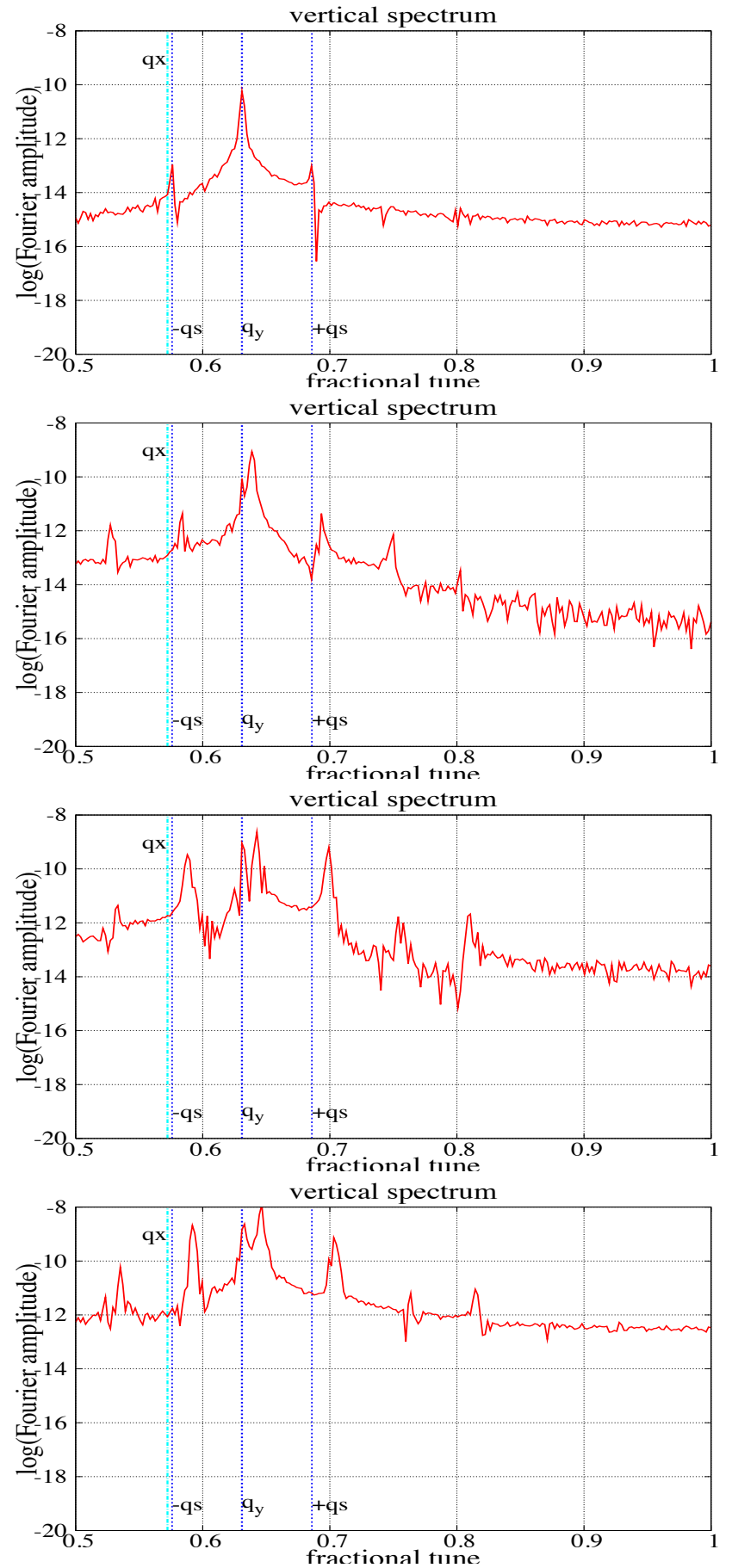

Figure 18: Spectrum of vertical bunch motion for varying cloud densities - from top to bottom $6 \times 10^{10}$, $3 \times 10^{11}, 6 \times 10^{11}$, and $8 \times 10^{11}$ electrons $/ \mathrm{m}^{3}$. 


\section{international linear collider}

3.4.3.4 RFA Modeling

A single RFA voltage scan, in which data is collected as a function of retarding voltage, measures the energy spectrum of the cloud electrons. In practice, however, it is a nontrivial task to map a data point from a voltage scan, namely the collector current, to cloud density in the middle of a vacuum chamber. Typically, this gap is bridged through the use of cloud simulation programs, like ECLOUD and POSINST, which track the motion of cloud particles during and after the passage of a bunch train, and a physics model of the RFA.

The simplest method for simulating the output of an RFA for a given set of beam conditions is postprocessing the output of one of these programs. These codes simulate the evolution of the cloud within the entire volume of the vacuum chamber by tracking macro-particles and can provide information for each collision of a macro-particle with the vacuum chamber wall. For collisions in the region of an RFA, one can perform a series of calculations on this output to determine the flux of cloud particles into the RFA. Note that by proceeding in this way one implicitly assumes that the presence of the RFA has no effect on the development of the cloud. This assumption is probably justified for a drift RFA, but is not typically true in a region with magnetic fields. In these regions, a detailed model of the RFA response needs to be included in the simulation.

The goal given a set of voltage scan data, is to find a set of simulation parameters that bring data and simulation into as close agreement as possible. We expect that the best-fit parameters obtained from this method will be close to the real values for the material under study. The parameters include

1. The peak true secondary electron yield

2. The low energy elastic yield

3. The re-diffused yield at infinity

4. The energy at which peak secondary production occurs

5. Quantum efficiency

6. The peak energy and width of the photoelectron energy distribution

7. The photon reflectivity

Figure 19 shows the results of the parameter finding method for a TiN-coated drift chamber. Overall there is good agreement between data and simulation for a wide variety of beam conditions. The simulation is based on an electron cloud generated with the POSINST code with the fit parameters. These types of fits are beginning to provide detailed insight into the differences between various coating materials that have been proposed to mitigate the $\mathrm{EC}$ in the DR vacuum chambers. 


\section{international linear collider}
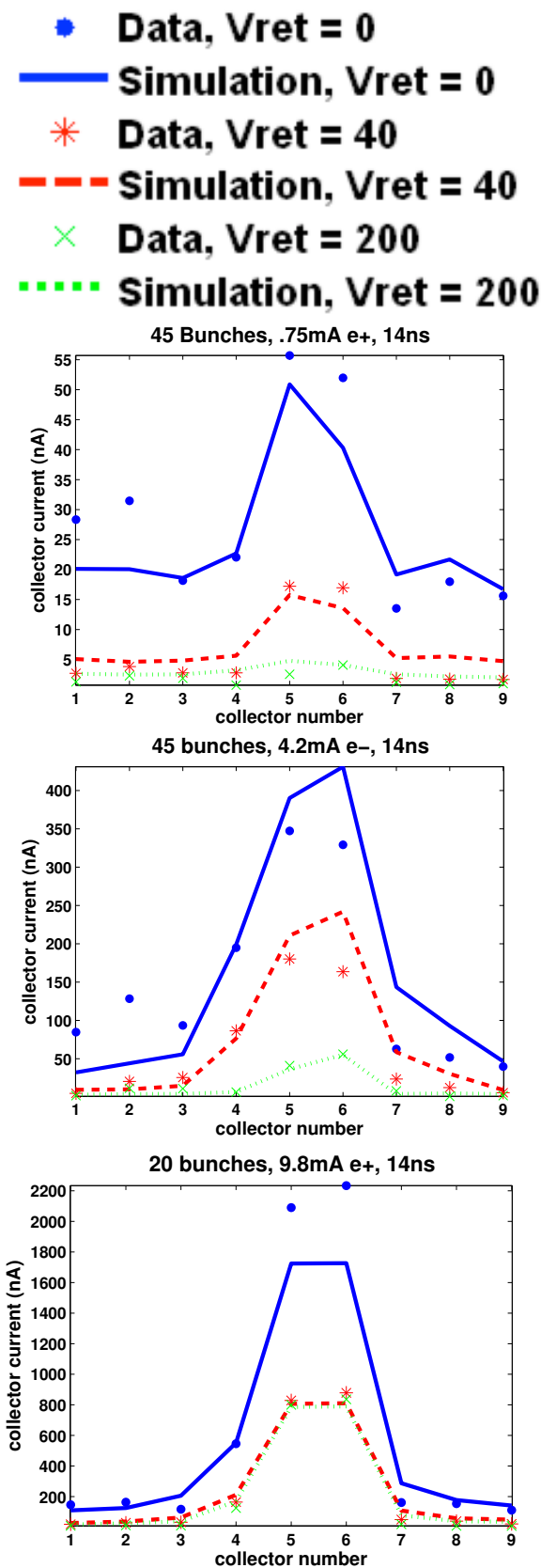
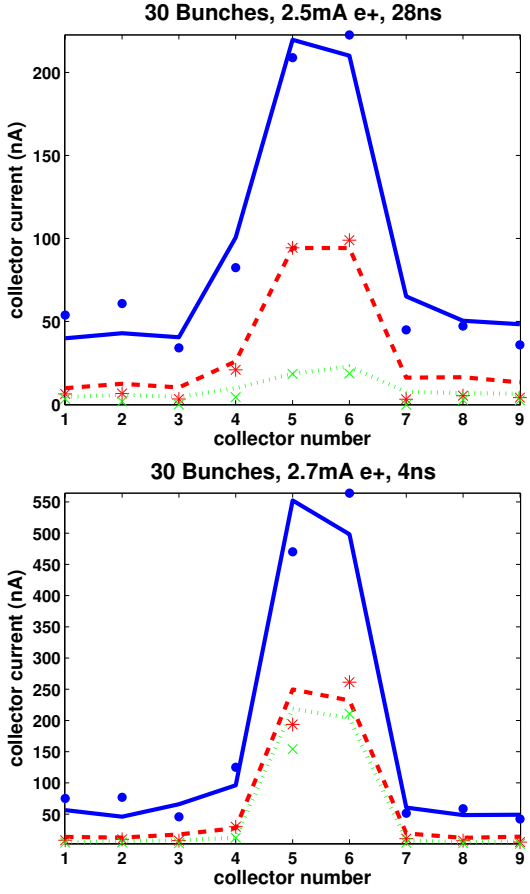

9 bunches, 3.8mA e-, 280ns
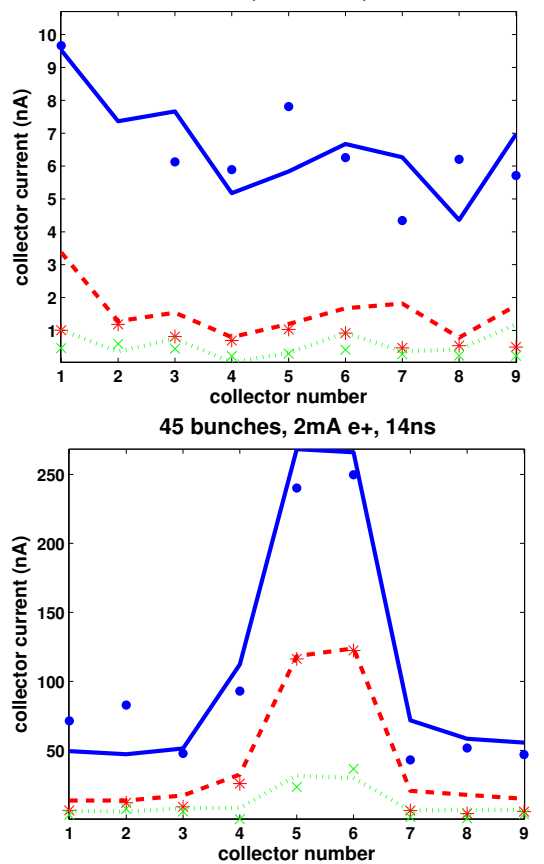

Figure 19: Comparison of RFA data and simulation, using best-fit parameters. The plots show the signal across the 9 RFA collectors at three different retarding voltages. All of these data sets were taken at beam energy $5.3 \mathrm{GeV}$. Note that for CESR, $\operatorname{ImA}=1.6 \times 10^{10}$ particles. The distribution of macro-particle wall collisions that are the input to the RFA simulation are generated with POSINST using the best-fit parameters. 


\section{international linear collider}

\subsubsection{Incorporation of CESRTA Results into the ILC Damping Rings Technical Design}

The results from the first 2.5 years of the CESRTA R\&D program are presently being integrated into the ILC DR technical design [57]. In particular, the observed efficacy of grooved chamber surfaces in the dipole dipoles as well as that of the clearing electrode in the high field wigglers provide confidence that practical EC mitigations can be prepared for the arc and wiggler straight regions of the ILC positron damping ring. The importance of EC mitigation in the DR quadrupole chambers has also been demonstrated. New coating technologies to suppress the SEY offer great promise, however, the longterm performance and durability of these coatings has yet to be established - this will be a subject of study during Phase II of the CESRTA program. Perhaps most importantly, the flexibility of CESR operations supports a systematic program of EC build-up and EC-induced beam dynamics studies. By benchmarking our physics models and simulations against these studies, our confidence in being able to make valid projections of the expected ILC positron damping performance has been significantly enhanced.

\subsubsection{Preliminary Recommendations of the ILC Electron Cloud Working Group}

A working group has been formed to evaluate the electron cloud effect and instability issues for the ILC positron damping ring and to recommend mitigation solutions. The Collaborating Institutions are: ANL, CERN, Cornell University, INFN, KEK, LBNL, SLAC. The first task of the working group was to compare the electron cloud effect for two different Damping Ring designs with $6.4 \mathrm{~km}$ and $3.2 \mathrm{~km}$ circumference, respectively, and to investigate the feasibility of the shorter damping ring with respect to the electron cloud build-up and related beam instabilities. We compared the instability thresholds and the electron cloud formation assuming $6 \mathrm{~ns}$ bunch spacing in both configurations, i.e., the same beam current. Both ring configurations were found to exhibit very similar performance. The risk associated with the adoption of the $3.2 \mathrm{~km}$ damping ring design, while maintaining the same bunch spacing, was deemed low and the $3.2 \mathrm{~km}$ ring was found an acceptable baseline design choice.

The main working group deliverables are the recommendations for the baseline and alternate solutions for the electron cloud mitigation in various regions of the ILC Positron Damping Ring. The preliminary mitigation recommendations for the ILC damping rings are the result of the working group discussions held during a number of workshops and regular online meetings. The working group met at Cornell University on October 13, 2010, as a satellite meeting to the ECLOUD10 Workshop held on October 812. The workshop was devoted to hearing the results of detailed studies of a range of mitigation options. Input from the workshop participants was included in the evaluation. The results of the evaluation were presented at the IWLC10 Workshop at CERN[58]. Table 6 provides a brief summary of the recommendations.

Table 6: Summary of baseline EC mitigation recommendations developed at the EC working group meeting held as part of the ECLOUD10 Workshop on October 13, 2010.

\begin{tabular}{|l|l|l|l|}
\hline Field Region & \multicolumn{2}{|c|}{ Baseline Mitigation Recommendation } & $\begin{array}{l}\text { Alternatives for Further } \\
\text { Investigation }\end{array}$ \\
\hline Drift* & TiN Coating & Solenoid Windings & NEG Coating \\
\hline Dipole & $\begin{array}{l}\text { Grooves with TiN } \\
\text { Coating }\end{array}$ & $\begin{array}{l}\text { Antechambers for power loads } \\
\text { and photoelectron control }\end{array}$ & $\begin{array}{l}\text { R\&D into the use of } \\
\text { clearing electrodes. }\end{array}$ \\
\hline Quadrupole* & TiN Coating & $\begin{array}{l}\text { R\&D into the use of } \\
\text { clearing electrodes or } \\
\text { grooves with TiN coating }\end{array}$ \\
\hline Wiggler & Clearing Electrodes & $\begin{array}{l}\text { Antechambers for power loads } \\
\text { and photoelectron control }\end{array}$ & $\begin{array}{l}\text { Grooves with TiN } \\
\text { Coating }\end{array}$ \\
\hline
\end{tabular}

* Where drift and quadrupole chambers are in arc or wiggler straight regions of the machine, the chambers will incorporate features of those sections, ie, antechambers for power loads and photoelectron control. 


\section{international linear collider}

\subsubsection{Personnel and Institutions Participating in the CESRTA Collaboration}

Personnel at Cornell with significant participation in the CESRTA effort include:

- Senior Staff: J. Alexander, S. Belomestnykh, M. Billing, J. Crittenden, G. Dugan, E. Fontes, S. Gray, S. Greenwald, D. Hartill, W. Hartung, J. Kandaswamy, D. Kreinick, Y. Li, X. Liu, R. Meller, M Palmer, D. Peterson, D. Rice, D. Rubin, D. Sagan, K. Sonnad, E. Smith, A. Temnykh, M. Tigner, and T. Wilksen;

- Technical Staff: S. Ball, J. Barley, L. Bartnik, D. Bougie, M. Carrier, S. Chapman, G. Codner, M. Comfort, C. Conolly, J. Conway, J. Dobbins, M. Forster, R. Gallagher, L. Hirshman, B. Johnson, A. Lyndaker, V. Medjidzade, T. Moore, R. Morey, T. O’Connell, S. Peck, M. Rendina, P. Revesz, N. Rider, D. Sabol, J. Savino, R. Seeley, J. Sexton, J. Sikora, K. Smolenski, M. Stedinger, C. Strohman, and D. Widger.

A key component of the research effort is the level of participation of collaborators. The CESRTA collaboration has involved over 50 senior staff from more than a dozen international laboratories and universities. The productiveness of the program would not have been possible without the support provided by these researchers. Table 7 summarizes the contributions from each group. This participation has included collaborative research efforts, in-kind contributions, direct financial support for specific experimental hardware, and personnel exchanges. Furthermore, the expertise represented by this collaboration in the areas of electron cloud measurements and modeling as well as low emittance tuning methods was crucial to the overall success of the research program.

A final group of participants in the research program consists of students. Table 8 lists student participants in the CESRTA program. This list includes graduate students (11), undergraduates from Cornell and collaborating institutions (16), and undergraduate participants in the NSF Research Experience for Undergraduates program (10), which is hosted by the Laboratory for Elementary-Particle Physics (LEPP) each summer. Thus student contributors represent a significant fraction of the total CESRTA research effort reflecting the emphasis at LEPP on training the next generation of physicists and engineers as part of the research program. 


\section{international linear collider}

Table 7: Research Staff from other institutions and their areas of involvement with the R\&D program.

\begin{tabular}{|c|c|c|}
\hline Collaborators & Institution & Participation \\
\hline K. Harkay & ANL & $\begin{array}{l}\text { Collaborative research and personnel exchange: electron cloud } \\
\text { measurements and simulations with a particular focus on } \\
\text { validation of the primary photoelectron models. }\end{array}$ \\
\hline R. Dowd & $\begin{array}{l}\text { Australian } \\
\text { Synchrotron }\end{array}$ & Collaborative research: low emittance tuning. \\
\hline W. Guo, S.Y. Zhang & $\mathrm{BNL}$ & Collaborative research: low emittance optics design. \\
\hline R. Holtzapple & CalPoly & $\begin{array}{l}\text { Collaborative research, in-kind contributions, and personnel } \\
\text { exchanges: instrumentation and beam dynamics measurements. }\end{array}$ \\
\hline D. Asner* & Carleton U. & Collaborative research: secondary electron yield measurements. \\
\hline $\begin{array}{l}\text { F. Antoniou, S. Calatroni, } \\
\text { F. Caspers, M. Gasior, } \\
\text { R. Jones, Y. Papaphilippou, } \\
\text { J. Pfingster, G. Rumolo, }\end{array}$ & CERN & $\begin{array}{l}\text { Collaborative research, in-kind contributions and personnel } \\
\text { exchanges: electron cloud measurements and simulations, beam } \\
\text { instrumentation, microwave transmission techniques, machine } \\
\text { stability, and beam dynamics studies. }\end{array}$ \\
\hline
\end{tabular}

Cockroft Collaborative research and personnel exchanges: low emittance

J. Jones, A. Wolski

M.C. Ross, C-Y. Tan,

R. Zwaska

Theo Demma

K. Kanazawa, K. Kubo,

K. Ohmi, K. Oide,

J. Flannagan, H. Sakai,

Y. Suetsugu, K. Shibata,

H. Tajima, M. Tobiyama,

J. Urakawa

J. Byrd, C. Celata, J. Corlett,

S. De Santis, M. Furman,

A. Jackson, R. Kraft,

D. Munson, G. Penn,

D. Plate, A. Rawlins,

M. Venturini, M. Zisman
A. Garfinkel
Purdue U.

D. Kharakh, J. Ng., M. Pivi, SLAC

L. Wang

L. Schächter

LBNL
INFN-LNF

(Frascati, IT)

KEK (Japan)

Collaborative research and personnel exchanges: electron cloud simulations with a particular focus on validation of the primary photoelectron models.

Collaborative research, in-kind support and personnel exchanges: electron cloud measurements and simulations, dipole and wiggler chambers for electron cloud suppression, secondary electron yield measurements.

Technion- Collaborative research and personnel exchanges: electron cloud Haifa (Israel) measurements and analysis.

\footnotetext{
* Now with Pacific Northwest National Laboratory, Hanford, WA
} 


\section{international linear collider}

Table 8: Student participants in the CESRTA Research Program

\begin{tabular}{|c|c|c|}
\hline Student & Institution & Participation \\
\hline \multicolumn{3}{|l|}{ Graduate Students } \\
\hline Joseph Calvey & Cornell & Electron cloud measurements \& simulations \\
\hline Nicholas Eggert & Cornell & xBSM development \\
\hline Michael Ehrlichman & Cornell & Intrabeam and Touschek scattering \\
\hline Richard Helms & Cornell & Accelerator design \\
\hline Walter Hopkins & Cornell & xBSM development \\
\hline Benjamin Kreis & Cornell & xBSM development \\
\hline James Shanks & Cornell & Low emittance tuning \\
\hline Jeremy Urban & Cornell & Accelerator design \\
\hline Yanay Yariv & Cornell & Low emittance tuning \\
\hline Laura Boon & Purdue & Electron cloud simulations \\
\hline Puneet Jain & KEK & Electron cloud simulations \\
\hline \multicolumn{3}{|c|}{ Undergraduate Students } \\
\hline Kaitlin Butler & Cornell & Electron cloud simulations \\
\hline Noah Kaminsky & Cornell & Electron cloud simulations \\
\hline Jin-Sung Kim & Cornell & Secondary electron yield measurements \\
\hline Zhidong Leong & Cornell & Electron cloud simulations \\
\hline Hongwan Liu & Cornell & xBSM development \\
\hline Jesse Livezey & Cornell & Electron cloud measurements \& simulations \\
\hline Junki Makita & Cornell & Electron cloud measurements \& simulations \\
\hline Michael McDonald & Cornell & xBSM development \\
\hline Gabriel Ramirez & Cornell & Electron cloud measurements \& simulations \\
\hline Steven Santos & Cornell & Electron cloud measurements \& simulations \\
\hline Robert Schwartz & Cornell & Electron cloud measurements \& simulations \\
\hline Siarhei Vishniakou & Cornell & Software support \\
\hline William Whitney & Cornell & Electron cloud measurements \\
\hline Heather Williams & Cornell & Beam dynamics studies \\
\hline Leah Fabrizzio & CalPoly & Instrumentation development \\
\hline Matthew Randazzo & CalPoly & Instrumentation/beam dynamics studies \\
\hline \multicolumn{3}{|c|}{$\begin{array}{l}\text { Research Experience for } \\
\text { Undergraduates }\end{array}$} \\
\hline Daniel Carmody & Carnegie Mellon & Electron cloud simulations \\
\hline Chris Cude & Indiana University & Electron cloud measurements \\
\hline Pauli Kehayias & Tufts & Beam dynamics simulation \\
\hline Matthew Lawson & Harvey Mudd College & Low emittance tuning \\
\hline Benjamin Carlson & Grove City College & Microwave measurements of electron cloud \\
\hline Daniel Gonnella & Clarkson & Intrabeam and Touschek Scattering \\
\hline Eric Wilkinson & Loyola & Electron cloud simulations \\
\hline Laurel Hales & University of Utah & Low emittance tuning \\
\hline Kenneth Hammond & Harvard University & Microwave measurements of electron cloud \\
\hline Neboysa Omcikus & American River College & Electron cloud simulations \\
\hline
\end{tabular}




\section{international linear collider}

\subsubsection{Ongoing Effort}

The collaboration is preparing complete technical documentation of the CESRTA research program with an anticipated publication date in the last half of 2011. During the next year we plan to explore electron cloud phenomena at lower beam emittances, especially instability thresholds and sub-threshold emittance growth. These studies will provide important updates to our projections of the ILC DR performance. We will also exploit the lower emittance regime to investigate other collective effects including intra-beam scattering, Touschek scattering, and the fast ion instability.This effort will require the development of instrumentation for measuring horizontal as well as vertical emittances. We will continue to refine tools and techniques for low emittance tuning, with the goal of real time monitoring and correction of orbit, coupling, and vertical dispersion. In addition, the extended operation of CESRTA will provide an opportunity to test the durability of mitigations. We also will continue to support the technical design effort for the ILC damping rings.

\subsubsection{DOE Budget Summary}

Table 9 summarizes the moneys from the US DOE that were expended as part of this research. Please note that additional funding from the US National Science Foundation was also used in support of the research effort.

Table 9: DOE Award Budget and Expenditures

\begin{tabular}{|c|c|c|c|c|c|}
\hline Budget & Salaries \& Fringe & M\&S & Travel M\&S & Indirect & $\begin{array}{c}\text { Total } \\
\text { Expenditures }\end{array}$ \\
\hline$\$ 5,284,000$ & $\$ 1,864,129$ & $\$ 2,208,424$ & $\$ 34,917$ & $\$ 1,176,530$ & $\$ 5,284,000$ \\
\hline
\end{tabular}




\section{international linear collider}

\section{Products Developed and Technology Transfer Activities}

\subsection{Publications and Workshop Contributions}

As of the conclusion of this award, over 60 workshop, journal, and technical publications had been prepared which were associated or relied on results obtained with the CESRTA R\&D Program.

\subsubsection{Publications}

The International Linear Collider: A Technical Progress Report, edited by E. Elsen, et al., ISBN: 978-3-935702-560, 2011. http://ilcdoc.linearcollider.org/record/32863/files/ilc_interim_report_2011-lores.pdf

C. M. Celata, "Electron Cloud Dynamics in the Cornell Electron Storage Ring Test Accelerator Wiggler," Phys. Rev. ST Accel. Beams 14, April 2011, 041003. Article (APS)

J. R. Calvey, J. A. Crittenden, G. F. Dugan, M. A. Palmer, M. Furman, K. Harkay, "Methods for Quantitative Interpretation of Retarding Field Analyzer Data," in Proceedings of the 2011 Particle Accelerator Conference, New York, NY, in press, PDF (PAC 2011/BNL)

S. De Santis, J. Byrd, J. Sikora, J. Calvey, J. Livezey, K. Sonnad, K. Hammond, “TE Wave Measurements of the Electron Cloud in a Dipole Magnetic Field," in Proceedings of the 2011 Particle Accelerator Conference, New York, $N Y$, in press, $\mathrm{PDF}$ (PAC 2011/BNL)

N. T. Rider, J. P. Alexander, J. A. Dobbins, M. G. Billing, R. E. Meller, M. A. Palmer, D. P. Peterson, C. R. Strohman, J. W. Flanagan, "Development of an X-Ray Beam Size Monitor with Single Pass Measurement Capability for CesrTA," in Proceedings of the 2011 Particle Accelerator Conference, New York, NY, in press, PDF (PAC 2011/BNL)

Joe Conway, Y. Li, X. Liu, V. Medjidzade, M. Palmer, "Implementation and Operation of Electron Cloud Diagnostics for CesrTA," in Proceedings of the 2011 Particle Accelerator Conference, New York, NY, in press, PDF (PAC 2011/BNL)

J. Kim, D. Asner, J. Conway, S. Greenwald, Y. Li, V. Medjidzade, T. Moore, M. Palmer, C. Strohman, "In-Situ Secondary Electron Yield Measurement System at CesrTA," in Proceedings of the 2011 Particle Accelerator Conference, New York, NY, in press, PDF (PAC 2011/BNL)

J. Shanks, M. Billing, R. Meller, M. Palmer, M. Rendina, D. Rubin, N. Rider, D. Sagan, C. Strohman, Y. Yanay, "Status of Low Emittance Tuning at CesrTA," in Proceedings of the 2011 Particle Accelerator Conference, New York, $N Y$, in press,

D. L. Kreinick, J. A. Crittenden, G. Dugan, M. A. Palmer, G. Ramirez, R. L. Holtzapple, M. Randazzo, M. A. Furman, M. Venturini, "Application of Coherent Tune Shift Measurements to the Characterization of Electron Cloud Growth," in Proceedings of the 2011 Particle Accelerator Conference, New York, NY, in press, PDF (PAC 2011/BNL)

K. G. Sonnad, K. Butler, G. Dugan, M. A. Palmer, M. T. F. Pivi, "Simulation of Electron Cloud Induced Instabilities and Emittance Growth for CesrTA," in Proceedings of the 2011 Particle Accelerator Conference, New York, $N Y$, in press,

J. A. Crittenden, D. C. Sagan, K. G. Sonnad, "Electron Cloud Modeling for the ILC Damping Rings," in Proceedings of the 2011 Particle Accelerator Conference, New York, NY, in press, PDF (PAC 2011/BNL) 


\section{international linear collider}

J. A. Crittenden, Y. Li, X. Liu, M. A. Palmer, J. P. Sikora, S. Calatroni, G. Rumolo, "Electron Cloud Modeling Results for Time-Resolved Shielded Pickup Measurements at CesrTA," in Proceedings of the 2011 Particle Accelerator Conference, New York, NY, in press, PDF (PAC 2011/BNL)

M. G. Billing, G. Dugan, M. J. Forster, R. E. Meller, M. A. Palmer, G. A. Ramirez, K. Sonnad, J. P. Sikora, H. A. Williams, R. L. Holtzapple, "Measurement Techniques to Characterize Instabilities Caused by Electron Clouds," in Proceedings of the 2011 Particle Accelerator Conference, New York, NY, in press, PDF (PAC 2011/BNL)

J. P. Sikora, M. G. Billing, J. A. Crittenden, Y. Li, M. A. Palmer, S. DeSantis, "Time Resolved Measurement of Electron Clouds at CesrTA Using Shielded Pickups," in Proceedings of the 2011 Particle Accelerator Conference, New York, NY, in press, PDF (PAC 2011/BNL)

Hyunchang Jin, Moohyun Yoon, Kazuhito Ohmi, John W. Flanagan, Mark A. Palmer, "Electron Cloud Effects in Cornell Electron Storage Ring Test Accelerator and International Linear Collider Damping Ring," Jpn. J. Appl. Phys. 50, February 2011, 026401. Article (JSAP)

\subsubsection{Publications}

D. Rubin, "CesrTA Program Overview," in Proceedings of ECLOUD 2010: 49th ICFA Advanced Beam Dynamics Workshop on Electron Cloud Physics, Ithaca, NY, edited by K. Smolenski, in press, Paper OPR06.

J. R. Calvey, J. Makita, M. A. Palmer, R. M. Schwartz, C. R. Strohman, S. Calatroni, G. Rumolo, K. Kanazawa, Y. Suetsugu, M. Pivi, L. Wang, "Electron Cloud Mitigation Investigations at CESR-TA," in Proceedings of ECLOUD 2010: 49th ICFA Advanced Beam Dynamics Workshop on Electron Cloud Physics, Ithaca, NY, edited by K.

Smolenski, in press, Paper MIT01.

G. Dugan, M. G. Billing, R. Meller, M. Palmer, G. A. Ramirez, J. Sikora, K. Sonnad, H. Williams, R. L. Holtzapple, "Studies of Electron-Cloud-Induced Beam Dynamics at CesrTA," in Proceedings of ECLOUD 2010: 49th ICFA Advanced Beam Dynamics Workshop on Electron Cloud Physics, Ithaca, NY, edited by K. Smolenski, in press, Paper DYN03.

K. Ohmi, H. Jin, Y. Susaki, "Electron Cloud Instability in Low Emittance Rings," in Proceedings of ECLOUD 2010: 49th ICFA Advanced Beam Dynamics Workshop on Electron Cloud Physics, Ithaca, NY, edited by K. Smolenski, in press, Paper DYN05.

Y. Li, X. Liu, V. Medjidzade, Joe Conway, Mark Palmer, "Implementation and Operation of Electron Cloud Diagnostics for CesrTA," in Proceedings of ECLOUD 2010: 49th ICFA Advanced Beam Dynamics Workshop on Electron Cloud Physics, Ithaca, NY, edited by K. Smolenski, in press, Paper PST01.

N. T. Rider, J. P. Alexander, M. G. Billing, C. Connoly, N. Eggert, E. Fontes, W. Hopkins, B. Kreis, A. Lyndaker, R. E. Meller, M. A. Palmer, D. P. Peterson, M. C. Rendina, P. Revesz, D. L. Rubin, J. Savino, R. Seeley, J. Shanks, C. R. Strohman, R. L. Holtzapple, J. W. Flanagan, "Bunch-by-Bunch Instrumentation Upgrades for CESR, Based on Requirements for the CESR Test Accelerator Research Program," in Proceedings of ECLOUD 2010: 49th ICFA Advanced Beam Dynamics Workshop on Electron Cloud Physics, Ithaca, NY, edited by K. Smolenski, in press, Paper PST02.

J. R. Calvey, J. A. Crittenden, G. F. Dugan, M. A. Palmer, K. Harkay, "Methods for Quantitative Interpretation of Retarding Field Analyzer Data," in Proceedings of ECLOUD 2010: 49th ICFA Advanced Beam Dynamics Workshop on Electron Cloud Physics, Ithaca, NY, edited by K. Smolenski, in press, Paper PST03.

J. P. Sikora, S. De Santis, K. Hammond, "TE Wave measurements at CesrTA," in Proceedings of ECLOUD 2010: 49th ICFA Advanced Beam Dynamics Workshop on Electron Cloud Physics, Ithaca, NY, edited by K. Smolenski, in press, Paper PST04. 


\section{international linear collider}

K. G. Sonnad, M. T. F. Pivi, J-L Vay, G. Rumolo, R. Tomas, F. Zimmermann, G. Franchetti, "An Update on Simulation of Beam Dynamics with Electron Cloud Effects," in Proceedings of ECLOUD 2010: 49th ICFA Advanced Beam Dynamics Workshop on Electron Cloud Physics, Ithaca, NY, edited by K. Smolenski, in press, Paper PST05.

Kenneth Hammond, John Sikora, Kiran G Sonnad, Seth Veitzer, "Effects of Reflections on TE-Wave Measurements of Electron Cloud Density," in Proceedings of ECLOUD 2010: 49th ICFA Advanced Beam Dynamics Workshop on Electron Cloud Physics, Ithaca, NY, edited by K. Smolenski, in press, Paper PST06.

M. G. Billing, G. Dugan, R. E. Meller, M. A. Palmer, G. A. Ramirez, J. P. Sikora, H. A. Williams, R. L. Holtzapple, "Techniques for Observing Beam Dynamical Effects Caused by the Presence of Electron Clouds," in Proceedings of ECLOUD 2010: 49th ICFA Advanced Beam Dynamics Workshop on Electron Cloud Physics, Ithaca, NY, edited by K. Smolenski, in press, Paper PST07.

G. Dugan, D. Sagan, "Synrad3D Photon Propagation and Scattering Simulation," in Proceedings of ECLOUD 2010: 49th ICFA Advanced Beam Dynamics Workshop on Electron Cloud Physics, Ithaca, NY, edited by K. Smolenski, in press, Paper PST08.

J. A. Crittenden, Y. Li, X, Liu, M. A. Palmer, J. P. Sikora, S. Calatroni, G. Rumolo, N. Omcikus, "Electron Cloud Modeling Results for Time-Resolved Shielded Pickup Measurements at CesrTA," in Proceedings of ECLOUD 2010: 49th ICFA Advanced Beam Dynamics Workshop on Electron Cloud Physics, Ithaca, NY, edited by K. Smolenski, in press, Paper PST09.

D. L. Kreinick, J. A. Crittenden, G. Dugan, Z. Leong, M. A. Palmer, R. L. Holtzapple, M. Randazzo, M. A. Furman, M. Venturini, "Using Coherent Tune Shifts to Evaluate Electron Cloud Effects on Beam Dynamics at CesrTA," in Proceedings of ECLOUD 2010: 49th ICFA Advanced Beam Dynamics Workshop on Electron Cloud Physics, Ithaca, NY, edited by K. Smolenski, in press, Paper PST10.

D. Rubin, D. Sagan, J. P. Shanks, Y. Yanay, "CesrTA Low Emittance Tuning," in Proceedings of ECLOUD 2010: 49th ICFA Advanced Beam Dynamics Workshop on Electron Cloud Physics, Ithaca, NY, edited by K. Smolenski, in press, Paper PST11.

J. Kim, D. Asner, J. Conway, S. Greenwald, Y. Li, V. Medjidzade, T. Moore, M. Palmer, C. Strohman, "In Situ SEY Measurements at CesrTA," in Proceedings of ECLOUD 2010: 49th ICFA Advanced Beam Dynamics Workshop on Electron Cloud Physics, Ithaca, NY, edited by K. Smolenski, in press, Paper PST12.

L. Wang, M. Pivi, "Trapping of Electron Cloud in ILC/CesrTA Quadrupole and Sextupole Magnets," in Proceedings of ECLOUD 2010: 49th ICFA Advanced Beam Dynamics Workshop on Electron Cloud Physics, Ithaca, $N Y$, edited by K. Smolenski, in press, Paper MOD05.

D. L. Rubin, M. Billing, R. Meller, M. Palmer, M. Rendina, N. Rider, D. Sagan, J. Shanks, C. Strohman, "Beam Based Measurement of Beam Position Monitor Electrode Gains," Phys. Rev. ST Accel. Beams 13, September 2010, 092802. Article (APS)

S. De Santis, J. M. Byrd, M. Billing, M. Palmer, J. Sikora, B. Carlson, "Characterization of Electron Clouds in the Cornell Electron Storage Ring Test Accelerator using TE-Wave Transmission," Phys. Rev. ST Accel. Beams 13, July 2010, 071002. Article (APS)

J. W. Flanagan, H. Fukuma, H. Ikeda, T. Mitsuhashi, G. S. Varner, J. P. Alexander, N. Eggert, W. H. Hopkins, B. Kreis, M. A. Palmer, D. P. Peterson, "Measurement of Low-Emittance Beam with Coded Aperture X Ray Optics at CesrTA," in Proceedings of the 2010 International Particle Accelerator Conference, Kyoto, Japan, 2010, p. 966968. PDF (JACoW) 


\section{international linear collider}

S. De Santis, J. Sikora, M. Billing, M. Palmer, B. Carlson, "TE Wave Measurements of the Electron Cloud in the Cesr-TA Ring," in Proceedings of the 2010 International Particle Accelerator Conference, Kyoto, Japan, 2010, p. 1188-1190. PDF (JACoW )

M. A. Palmer, M. G. Billing, R. E. Meller, M. C. Rendina, N. T. Rider, D. L. Rubin, J. Shanks, C. R. Strohman, R. L. Holtzapple, "CESR Beam Position Monitor System Upgrade for CesrTA and CHESS Operations," in Proceedings of the 2010 International Particle Accelerator Conference, Kyoto, Japan, 2010, p. 1191-1193. PDF (JACoW )

D. P. Peterson, J. P. Alexander, C. Conolly, N. Eggert, E. Fontes, W. Hopkins, B. Kries, A. Lyndaker, M. McDonald, M. Palmer, M. Rendina, P. Revesz, N. Rider, J. Savino, R. Seeley, J. W. Flanagan, "CesrTA x-Ray Beam Size Monitor Operation," in Proceedings of the 2010 International Particle Accelerator Conference, Kyoto, Japan, 2010, p. 1194-1196. PDF (JACoW )

M. G. Billing, G. Dugan, R. Meller, M. Palmer, M. Rendina, N. Rider, J. Sikora, C. Strohman, R. L. Holtzapple, "Techniques for Observation of Beam Dynamics in the Presence of an Electron Cloud," in Proceedings of the 2010 International Particle Accelerator Conference, Kyoto, Japan, 2010, p. 1197-1199. PDF (JACoW )

M. A. Palmer, J. P. Alexander, M. G. Billing, J. R. Calvey, C. Conolly, J. A. Crittenden, J. A. Dobbins, G. F. Dugan, N. Eggert, E. Fontes, M. J. Forster, R. E. Gallagher, S. W. Gray, S. Greenwald, D. L. Hartill, W. H. Hopkins, D. L. Kreinick, B. Kreis, Z. Leong, Y. Li, X. Liu, J. A. Livezey, A. Lyndaker, J. Makita, M. McDonald, V. Medjidzade, R. E. Meller, T. I. O'Connell, S. B. Peck, D. P. Peterson, G. Ramirez, M. C. Rendina, P. Revesz, D. H. Rice, N. T. Rider, D. L. Rubin, D. C. Sagan, J. Savino, R. M. Schwartz, R. Seeley, J. Sexton, J. Shanks, J. P. Sikora, C. R. Strohman, H. Williams, K. C. Harkay, R. Dowd, W. Guo, R. L. Holtzapple, L. Fabrizzo, M. Randazzo, D. Asner, E. Smith, F. Antoniou, S. Calatroni, M. Gasior, R. Jones, Y. Papaphilippou, J. Pfingstner, G. Rumolo, H. Schmickler, M. Taborelli, D. Gonnella, J. Jones, A. Wolski, M. C. Ross, C. Y. Tan, R. Zwaska, B. Carlson, T. Demma, J. Flanagan, P. Jain, K. Kanazawa, K. Kubo, K. Ohmi, H. Sakai, K. Shibata, Y. Suetsugu, M. Tobiyama, J. Byrd, C. M. Celata, J. Corlett, S. De Santis, M. Furman, A. Jackson, R. Kraft, D. Munson, G. Penn, D. Plate, M. Venturini, E. L. Wilkinson, L. Boon, A. F. Garfinkel, D. Kharakh, J. Ng, M. T. F. Pivi, L. Wang, L. Schächter, "Electron Cloud at Low Emittance in CesrTA," in Proceedings of the 2010 International Particle Accelerator Conference, Kyoto, Japan, 2010, p. 1251-1255. PDF (JACoW)

L. Wang, X. Huang, M. Pivi, "Electron Trapping in Wiggler and Quadrupole Magnets of CesrTA," in Proceedings of the 2010 International Particle Accelerator Conference, Kyoto, Japan, 2010, p. 1892-1894. PDF (JACoW )

J. R. Calvey, C. M. Celata, J. A. Crittenden, G. F. Dugan, S. Greenwald, Z. Leong, J. Livezey, M. A. Palmer, M. Furman, M. Venturini, K. Harkay, "CesrTA Retarding Field Analyzer Modeling Results," in Proceedings of the 2010 International Particle Accelerator Conference, Kyoto, Japan, 2010, p. 1970-1972. PDF (JACoW )

J. R. Calvey, Y. Li, J. A. Livezey, J. Makita, R. E. Meller, M. A. Palmer, R. M. Schwartz, C. R. Strohman, K. Harkay, S. Calatroni, G. Rumolo, K. Kanazawa, Y. Suetsugu, M. Pivi, L. Wang, "CesrTA Retarding Field Analyzer Measurements in Drifts, Dipoles, Quadrupoles and Wigglers," in Proceedings of the 2010 International Particle Accelerator Conference, Kyoto, Japan, 2010, p. 1973-1975. PDF (JACoW )

J. A. Crittenden, J. R. Calvey, G. F. Dugan, D. L. Kreinick, Z. Leong, J. A. Livezey, M. A. Palmer, D. L. Rubin, D. C. Sagan, K. Harkay, R. L. Holtzapple, M. A. Furman, G. Penn, M. Venturini, M. Pivi, L. Wang, "Progress in Studies of Electron-cloud-induced Optics Distortions at CesrTA," in Proceedings of the 2010 International Particle Accelerator Conference, Kyoto, Japan, 2010, p. 1976-1978. PDF (JACoW)

M. T. F. Pivi, L. Wang, T. Demma, S. Guiducci, Y. Suetsugu, K. Shibata, K. Ohmi, G. Dugan, M. Palmer, J. A. Crittenden, K. Harkay, L. Boon, M. A. Furman, M. Venturini, C. Celata, O. B. Malyshev, I. Papaphilippou, "Recommendation for the Feasibility of More Compact LC Damping Rings," in Proceedings of the 2010 International Particle Accelerator Conference, Kyoto, Japan, 2010, p. 3578-3580. PDF (JACoW ) 


\section{international linear collider}

J. P. Shanks, D. Rubin, D. Sagan, "CesrTA Low Emittance Tuning," in Proceedings of the 2010 International Particle Accelerator Conference, Kyoto, Japan, 2010, p. 4620-4622. PDF (JACoW )

J. Sikora, Y. Li, M. Palmer, S. De Santis, D. Munson, "A Shielded Pick-Up Detector for Electron Cloud Measurements in the Cesr-TA Ring," in Proceedings of BIW 2010: Fourteenth Beam Instrumentation Workshop, Santa Fe, NM, edited by Clay Dillingham, Joe Chew, 2010, p. 345-349. PDF (JACoW )

\subsubsection{Publications}

G. F. Dugan, M. A. Palmer, D. L. Rubin, "ILC Damping Rings R\&D at CesrTA,” in ICFA Beam Dynamics Newsletter, International Committee on Future Accelerators, No. 50, edited by J. Urakawa, December 2009, Section 3.1, p. 11-33. Newsletter (ICFA/FNAL, PDF, 10 MBytes)

Benjamin Carlson, Michael Billing, Mark Palmer, John Sikora, "Implementation and Comparison of Electron Cloud Measurements at the Cornell Electron Storage Ring," REU Report, LEPP, Cornell University, Ithaca, NY, 2009, PDF E-Print (LEPP)

Y. Li, X. Liu, V. Medjidzade, J. Savino, D. Rice, D. Rubin, M. Palmer, "CesrTA Vacuum System Modifications," in Proceedings of the 2009 Particle Accelerator Conference, Vancouver, BC, 2009, p. 357-359. PDF (JACoW )

D. Rubin, D. Sagan, J. Shanks, "CesrTA Layout and Optics," in Proceedings of the 2009 Particle Accelerator Conference, Vancouver, BC, 2009, p. 2751-2753. PDF (JACoW)

J. P. Shanks, M. G. Billing, S. S. Chapman, M. J. Forster, S. B. Peck, D. L. Rubin, D. Sagan, J. W. Sexton, "CesrTA Low-Emittance Tuning---First Results," in Proceedings of the 2009 Particle Accelerator Conference, Vancouver, $B C, 2009$, p. 2754-2756. PDF (JACoW )

J. R. Calvey, J. A. Crittenden, G. Dugan, M. A. Palmer, C. M. Celata, "Electron Cloud Modeling Considerations at CesrTA," in Proceedings of the 2009 Particle Accelerator Conference, Vancouver, BC, 2009, p. 3306-3308. PDF (JACoW )

J. Alexander, C. Conolly, J. Flanagan, E. Fontes, W. Hopkins, B. Kreis, H. Liu, A. Lyndaker, D. Peterson, P. Revesz, J. Savino, R. Seeley, M. Palmer, "CesrTA X-Ray Beam Size Monitor Design," in Proceedings of the 2009 Particle Accelerator Conference, Vancouver, BC, 2009, p. 3503-3504. PDF (JACoW )

J. Alexander, W. Hopkins, B. Kreis, H. Liu, D. Peterson, J. Flanagan, "First Results from the CesrTA X-Ray Beam Size Monitor," in Proceedings of the 2009 Particle Accelerator Conference, Vancouver, BC, 2009, p. 35053506. PDF (JACoW)

Y. Li, M. G. Billing, S. Greenwald, T. I. O'Connell, M. A. Palmer, J. P. Sikora, E. N. Smith, K. W. Smolenski, J. N. Corlett, R. Kraft, D. V. Munson, D. W. Plate, A. W. Rawlins, K. Kanazawa, Y. Suetsugu, M. T. F. Pivi, "Design and Implementation of CesrTA Superconducting Wiggler Beampipes with Thin Retarding Field Analyzers," in Proceedings of the 2009 Particle Accelerator Conference, Vancouver, BC, 2009, p. 3507-3509. PDF (JACoW )

M. A. Palmer, M. G. Billing, J. R. Calvey, G. W. Codner, S. Greenwald, Y. Li, X. Liu, J. A. Livezey, R. E. Meller, R. M. Schwartz, J. P. Sikora, C. R. Strohman, W. S. Whitney, T. Wilksen, "Design, Implementation and First Results of Retarding Field Analyzers Developed for the CesrTA Program," in Proceedings of the 2009 Particle Accelerator Conference, Vancouver, BC, 2009, p. 3510-3512. PDF (JACoW )

J. W. Flanagan, H. Fukuma, S. Hiramatsu, H. Ikeda, K. Kanazawa, T. Mitsuhashi, J. Urakawa, G. S. Varner, J. P. Alexander, W. H. Hopkins, B. Kreis, M. A. Palmer, D. P. Peterson, "Performance of Coded Aperture X-Ray Optics with Low Emittance Beam at CesrTA," in Proceedings of the 2009 Particle Accelerator Conference, Vancouver, $B C, 2009$, p. 3561-3563. PDF (JACoW ) 


\section{international linear collider}

S. De Santis, J. Byrd, J. Sikora, M. Billing, "The TE Wave Transmission Method for Electron Cloud Measurements at Cesr-TA," in Proceedings of the 2009 Particle Accelerator Conference, Vancouver, BC, 2009, p. 3606-

3608. PDF (JACoW)

M. A. Palmer, J. Alexander, M. Billing, J. Calvey, S. Chapman, G. Codner, C. Conolly, J. Crittenden, J. Dobbins, G. Dugan, E. Fontes, M. Forster, R. Gallagher, S. Gray, S. Greenwald, D. Hartill, W. Hopkins, J. Kandaswamy, D. Kreinick, Y. Li, X. Liu, J. Livezey, A. Lyndaker, V. Medjidzade, R. Meller, S. Peck, D. Peterson, M. Rendina, P. Revesz, D. Rice, N. Rider, D. Rubin, D. Sagan, J. Savino, R. Seeley, J. Sexton, J. Shanks, J. Sikora, K. Smolenski, C. Strohman, A. Temnykh, M. Tigner, W. Whitney, H. Williams, S. Vishniakou, T. Wilksen, K. Harkay, R. Holtzapple, E. Smith, J. Jones, Y. He, M. Ross, C. Y. Tan, R. Zwaska, J. Flanagan, P. Jain, K. Kanazawa, K. Ohmi, H. Sakai, K. Shibata, Y. Suetsugu, J. Byrd, C. M. Celata, J. Corlett, S. De Santis, M. Furman, A. Jackson, R. Kraft, D. Munson, G. Penn, D. Plate, A. Rawlins, M. Venturini, M. Zisman, D. Kharakh, M. Pivi, L. Wang, "The Conversion and Operation of the Cornell Electron Storage Ring as a Test Accelerator (CesrTA) for Damping Rings Research and Development," in Proceedings of the 2009 Particle Accelerator Conference, Vancouver, BC, 2009, p. 4200-4204. PDF (JACoW )

J. Calvey, J. A. Crittenden, G. Dugan, S. Greenwald, D. Kreinick, J. A. Livezey, M.A. Palmer, D. Rubin, K. C. Harkay, P. Jain, K. Kanazawa, Y. Suetsugu, C. M. Celata, M. Furman, G. Penn, M. Venturini, M. T. F. Pivi, L.Wang, "Simulations of Electron-Cloud Current Density Measurements in Dipoles, Drifts and Wigglers at CesrTA," in Proceedings of the 2009 Particle Accelerator Conference, Vancouver, BC, 2009, p. 4628-4630. PDF (JACoW )

J. A. Crittenden, J. R. Calvey, G. Dugan, J. A. Livezey, D. L. Kreinick, M. A. Palmer, D. L. Rubin, K. Harkay, R. L. Holtzapple, K. Ohmi, M. A. Furman, G. Penn, M. Venturini, M.T.F. Pivi, L. Wang, "Studies of the Effects of Electron Cloud Formation on Beam Dynamics at CesrTA," in Proceedings of the 2009 Particle Accelerator Conference, Vancouver, BC, 2009, p. 4631-4633. PDF (JACoW )

\subsubsection{Publications}

J. W. Flanagan, H. Fukuma, S. Hiramatsu, H. Ikeda, K. Kanazawa, T. Mitsuhashi, J. Urakawa, G. S. Varner, J. P. Alexander, M. A. Palmer, "X-ray Monitor Based on Coded-aperture Imaging for KEKB Upgrade and ILC Damping Ring," in Proceedings of the 2008 European Particle Accelerator Conference, Genoa, Italy, 2008, p. 10291031. PDF (JACoW)

\subsubsection{Earlier Publications Associated Specifically with this Project}

M. A. Palmer, J. Alexander, M. Ehrlichman, D. Hartill, R. Helms, D. Rice, D. Rubin, D. Sagan, L. Schächter, J. Shanks, M. Tigner, J. Urban, "Plans for Utilizing the Cornell Electron Storage Ring as a Test Accelerator for ILC Damping Ring Research and Development," in Proceedings of the 2007 Particle Accelerator Conference, Albuquerque, NM, edited by C. Petit-Jean-Genaz, 2007, p. 42-44. PDF (JACoW )

M. A. Palmer, G. Codner, D. Rice, L. Schächter, E. Tanke, R. Holtzapple, J. Kern, "Electron Cloud Studies at CESR-c and Cesr-TA," in Proceedings of ECLOUD 2007: International Workshop on Electron-Cloud Effects, Daegu, Korea, edited by H. Fukuma, E. S. Kim, K. Ohmi, KEK Proceedings 2007-10, 2007, p. 108-113. Preprint (LEPP) Proceedings (CHEP/KNU, PDF, 34 MBytes)

M. A. Palmer, R. W. Helms, D. L. Rubin, D. C. Sagan, J. T. Urban, M. Ehrlichman, "The Proposed Conversion of CESR to an ILC Damping Ring Test Facility," in Proceedings of the 2006 European Particle Accelerator Conference, Edinburgh, Scotland, 2006, p. 891-893. PDF (JACoW ) 


\section{international linear collider}

\subsection{Web Sites}

Two principal web sites have been maintained as part of this project:

The CESRTA Collaboration Wiki Page

https://wiki.lepp.cornell.edu/ilc/bin/view/Public/CesrTA/WebHome

The ILC Damping Rings Wiki Page

https://wiki.lepp.cornell.edu/ilc/bin/view/Public/DampingRings/WebHome

The CESRTA Wiki site provides a central access point for the collaboration, where meeting notes, presentations and general information relevant to the research program are maintained. The ILC Damping Rings Wiki site has been the standard location for maintaining ILC Damping Rings information since the time when the ILC Reference Design Report (RDR) was being prepared. It contains detailed lattice information, a database of damping rings components specified in the RDR, and an archive of teleconference meeting presentations.

In addition to the above two web sites, archival web sites for the 3 workshops hosted over the course of this grant are also being maintained on LEPP servers. The workshop web sites contain presentations summarizing key results as well as working group recommendations and prioritizations for the CESRTA research program, which are of ongoing relevance. These web sites are available at:

ILCDR08 (July 8-11, 2008) -

https://wiki.lepp.cornell.edu/ilc/bin/view/Public/DampingRings/ILCDR08/

CTA09 (June 25-26, 2009) -

https://wiki.lepp.cornell.edu/ilc/bin/view/Public/DampingRings/CTA09/WebHome

ECLOUD10 (October 8-12, 2010) -

http://www.lepp.cornell.edu/Events/ECLOUD10/

\subsection{Networks and Collaborations Fostered}

The principal collaboration fostered as part of this grant has been the CESRTA Collaboration. This collaboration is composed of researchers from around the world with interests in:

- Electron cloud physics and its suppression in future high intensity particle accelerators;

- Advanced instrumentation techniques;

- Machine correction and tuning tools for future accelerators, which will be operating in the ultra low emittance regime.

As has been described in Sect. 3.4.6, the collaboration has involved over 50 senior researchers from over a dozen institutions around the world.

Phase I of the CESRTA research program has been supported under the umbrella of the ILC Global Design Effort (GDE). Work conducted throughout the program has been primarily directed towards providing critical $R \& D$ results to be incorporated in the final ILC Technical Design Report, which is the principal deliverable of the GDE.

An outgrowth of interactions between the ILC GDE and the Compact Linear Collider (CLIC) group based at CERN, has been the formation of a CLIC-ILC Joint Working Group on Damping Rings. Research efforts, past and future, at CESRTA have been closely coordinated with this group which encompasses the majority of the world-wide research community interested in damping rings. The research capabilities of CESR in its current damping ring configuration are broadly applicable to a range of experimental questions and technical development required for any of the proposed future damping rings. 


\section{international linear collider}

Two elements of the CESRTA Collaboration deserve further mention because they focus on machines whose upgrade plans will take place on timescales significantly shorter than the construction timescale envisioned for a possible linear collider damping ring. We have worked closely with the SuperKEKB design team and the Project $\mathrm{X}$ researchers evaluating EC issues for a Project X upgrade of the Fermilab Main Injector (MI). The results of our EC mitigation studies are relevant to both of these projects and the results of our research program have been incorporated into planning for the SuperKEKB positron ring vacuum system. In addition, the SuperKEKB beam size monitors are closely related to the technology that has been developed for the CESRTA xBSM. The effort at Cornell has both benefitted from technology developments for SuperKEKB as well as providing a crucial venue for prototyping new hardware being proposed for use in SuperKEKB.

A final collaboration, that has been directly supported during the latter portion of this grant, is the Low Emittance Rings Collaboration which developed out of the CLIC-ILC Joint Working Group on Damping Rings. The stated goal of this collaboration is to:

"bring together experts from the scientific communities working on low emittance lepton rings. This includes damping rings, test facilities for linear colliders, B-factories and electron storage rings. The theme will be common beam dynamics and technology challenges for producing and controlling ultra low emittance beams and the participants will benefit from the experience of colleagues who have designed, commissioned and operated such rings."

Thus the CESRTA research program has a great deal to contribute as well as to gain from the expertise and topical coverage of this collaboration.

\subsection{Technologies and Techniques Developed}

As is clear from the preceding section, the technologies and techniques that have been developed and applied through the course of the CESRTA R\&D program are of significant interest to the accelerator physics community. This section briefly summarizes the most significant developments of the CESRTA program that are being or can soon be applied at other accelerators.

\subsubsection{Low Emittance Tuning Techniques}

Ring correction at CESR relies heavily on measurements of resonantly excited beams (see Sect. 3.3), which, when coupled with a precision turn-by-turn BPM system, have now been demonstrated to give a reliable route to ultra low emittance optics correction of the ring. These techniques offer the potential for rapid and effective ring correction on timescales much shorter than are typical with storage rings. They also offer the possibility of real-time ring monitoring/correction using probe bunches as long as the BPM system is capable of measuring individual bunches on a turn-by-turn basis. Preliminary discussions about testing these techniques outside of Cornell have taken place during Phase I of the CESRTA program. Plans for Phase II of the program (which has now received funding) include further refinement of these techniques and the possibility of collaborating on their application at other facilities.

\subsubsection{X-Ray Beam Size Monitor with Single Pass Bunch Measurement Capability}

The xBSM system provides high-resolution measurement capability for ultra low emittance beams. As discussed in Sect. 4.3, the CESRTA system is serving as a test-bed for prototyping key portions of the technology that will be deployed at SuperKEKB. A unique feature of this system is its ability to acquire bunch-by-bunch data on a turn-by-turn basis, thus making it a device which is uniquely suited for the study of beam dynamics effects in the bunch trains of high intensity lepton storage rings. This makes it of interest both to the high energy physics and light source communities. Phase I of the CESRTA program has provided the initial demonstration and application of this type of device. We expect to further develop it, with the hope of eventually producing a new instrument, which can be made available for community-wide applications, during the recently funded Phase II program. 


\section{international linear collider}

4.4.3 Electron Cloud Mitigation Techniques

The electron cloud mitigation techniques, which have been studied during Phase I of the CESRTA program, are already being incorporated into planning for the SuperKEKB upgrade as well as the mitigation plan for the ILC positron DR. Preliminary documentation on these studies has been presented in a number of workshop and conference proceedings and more detail will appear in the CESRTA Phase I Report, which should be released in the last half of 2011. These results represent a key contribution to our understanding of how to control the EC in future high intensity accelerators.

\subsection{CESRTA Research Data}

Data acquired as part of the research program is being maintained in an archive available to all collaboration members. The experimental data archive can be found at:

https://wiki.lepp.cornell.edu/ilc/bin/view/Public/CesrTA/CesrTAData

Because this data represents one of the most detailed collections of electron cloud measurements in existence, which allows us to systematically characterize the build-up of the EC over a broad range of operating conditions, the long-term availability of the data for analysis is an issue of particular significance. Multiple archives of the raw data are being maintained on high reliability servers at LEPP. The data has also been further backed up to tape archives. Finally, we are also beginning to deploy analyzed archives for use in higher-level experimental analyses. We expect to maintain all of these archives for ongoing research use throughout the course of the extended CESRTA R\&D program. 


\section{international linear collider}

\section{References}

[1] M.A. Palmer, et al., "Electron Cloud at Low Emittance in CESRTA", Proceedings of IPAC'10, Kyoto, Japan (2010) and references therein.

[2] The CESRTA Collaboration includes senior researchers from the following institutions: ANL, Australian Synchrotron, BNL, California Polytechnic State Univ., Carleton Univ., CERN, the Cockroft Institute, FNAL, INFN-LNF, KEK, LBNL, Purdue University, SLAC and Technion-Haifa.

[3] http://www.lns.cornell.edu/Events/ECLOUD10/WebHome.html

[4] https://wiki.lepp.cornell.edu/ilc/bin/view/Public/DampingRings/WebHome Working_Group_on_Electron_Cloud

[5] M.T.F. Pivi, et al., "Recommendation for the Feasibility of More Compact LC Damping Rings", Proceedings of IPAC'10, Kyoto, Japan (2010).

[6] http://www.Ins.cornell.edu/Events/ECLOUD10/ILCDampingRingsSatelliteMeeting.html

[7] M.A. Palmer, et al., "The Conversion and Operation of the Cornell Electron Storage Ring as a Test Accelerator (CESRTA) for Damping Rings Research and Development", Proc. of PAC09, Vancouver, Canada (2009), and references therein.

[8] Y. Li, et al., "CesrTA Vacuum System Modifications", Proceedings of PAC09, Vancouver, British Columbia, Canada (2009).

[9] D. Rice, "CESR-c: A Wiggler-dominated Collider", Proceedings of PAC07, Albuquerque, New Mexico, USA (2007).

[10] M.A. Palmer, et al., "CESR Beam Position Monitor System Upgrade for CesrTA and CHESS Operations", Proceedings of IPAC10, Kyoto, Japan (2010).

[11] J. Alexander, et al., "CesrTA X-Ray Beam Size Monitor Design", Proceedings of PAC09, Vancouver, British Columbia, Canada (2009).

[12] M.A. Palmer, et al., "Design, Implementation and First Results of Retarding Field Analyzers Developed for the CesrTA Program", Proceedings of PAC09, Vancouver, British Columbia, Canada (2009).

[13] Y. Li, et al., "Design and Implementation of CesrTA Superconducting Wiggler Beampipes with Thin Retarding Field Analyzers", Proceedings of PAC09, Vancouver, British Columbia, Canada (2009).

[14] S. De Santis, et al. "The TE Wave Transmission Method for Electron Cloud Measurements at CesrTA", Proceedings of PAC09, Vancouver, British Columbia, Canada (2009).

[15] M.G. Billing, et al., "Techniques for Observation of Beam Dynamics in the Presence of an Electron Cloud", Proceedings of IPAC'10, Kyoto, Japan (2010).

[16] D. Rubin, et al., "CesrTA Layout and Optics", Proceedings of PAC09, Vancouver, British Columbia, Canada (2009).

[17] D. Sagan, "BMAD: A Relativistic Charged Particle Simulation Library", Nuclear Instruments and Methods in Physics Research, Volume A 558, March 2006, pp. 356-359

[18] D. Sagan and J. A. Crittenden and D. Rubin and E. Forest, "A Magnetic Field Model for Wigglers and Undulators",Proceedings of the 2003 Particle Accelerator Conference, Portland, OR, 2003, pp. 1023-1025 "http://accelconf.web.cern.ch/AccelConf/p03/PAPERS/MPPG006.PDF",

[19] R. Helms, PhD Thesis, January 2008

[20] D. Sagan and R. Meller and R. Littauer and D. Rubin,"Betatron Phase and Coupling Measurements at the Cornell Electron/Positron Storage Ring", Phys. Rev. ST Accel. Beams, 3 (092801), Sep, 2000

[21] D. Rubin, M.Billing, R. Meller, M. Palmer, M. Rendina, N.Rider, D. Sagan, J.Shanks, C. Strohman, "Beam based measurement of beam position monitor electrode gains", Phys. Rev. ST Accel. Beams, 13, 092802 (2010)

[22] J.R. Calvey, et al., "CesrTA Retarding Field Analyzer Measurements in Drifts, Dipoles, Quadrupoles and Wigglers", Proceedings of IPAC"10, Kyoto, Japan (2010).

[23] J.R. Calvey, et al., "Electron Cloud Mitigation Investigations at CesrTA", to be published in the proceedings of the ECLOUD`10 workshop, Ithaca, New York, USA (2010).

[24] J.R. Calvey, et al., "CesrTA Retarding Field Analyzer Modeling Results", Proceedings of IPAC'10, Kyoto, Japan (2010).

[25] J.R. Calvey, et al., "Methods for Quantitative Interpretation of Retarding Field Analyzer Data", to appear in the Proceedings of the ECLOUD10 Workshop, Ithaca, New York, USA (2010).

[26] M. T.F. Pivi, et al, "Secondary Electron Yield Measurements and Groove Chambers Update Tests in the PEPII Beam Line", 691, proc. of EPAC08, Genova, Italy, 2008.

[27] M. T.F. Pivi, et al., "A New Chicane Experiment in PEP-II to Test Mitigations of the Electron Cloud Effect for Linear Colliders”, 688, proc. of EPAC08, Genova, Italy, 2008. 


\section{international linear collider}

[28] S. De Santis et al., "Characterization of Electron Clouds in the Cornell Electron Storage Ring Test Accelerator using TE-Wave Transmission”, Phys. Rev. ST Accel. Beams 13:071002 (2010).

[29] G. Penn, J-L. Vay, "Theoretical Studies of TE-Wave Propagation as a Diagnostic for Electron Cloud", Proceedings of IPAC'10, Kyoto, Japan (2010).

[30] K. Sonnad, et al., "Simulations Using VORPAL on the Effect of Imperfections and Nonuniformities in TE Wave Propagation Through Electron Clouds", to appear in the Proceedings of the ECLOUD10 Workshop, Ithaca, NY, USA (2010).

[31] S. Veitzer, et al., "Modeling Electron Cloud Buildup and Microwave Diagnostics Using Vorpal", to appear in the Proceedings of the ECLOUD10 Workshop, Ithaca, NY, USA (2010).

[32] J. Crittenden, et al., "Electron Cloud Modeling Results for Time-resolved Shielded Pickup Measurements at CesrTA", to appear in the Proceedings of the ECLOUD10 Workshop, Ithaca, NY, USA (2010).

[33] Y. Suetsugu, et al., "Demonstration of Electron Clearing Effect by Means of Clearing Electrodes and Groove Structures in High-Intensity Positron Ring", proc. of PAC09, Vancouver, Canada (2009).

[34] Y. Suetsugu, et al., "Demonstration of electron clearing effect by means of a clearing electrode in highintensity positron ring," Nucl.Instrum. Methods A598 (2009) 372.

[35] Y. Suetsugu, et al, "Beam Tests of a Clearing Electrode for Electron Cloud Mitigation at KEKB Positron Ring”, Proceedings of IPAC'10, Kyoto, Japan, 2010.

[36] C. Yin Vallgren, et al., "Amorphus Carbon Coatings for Mitigation of Electron Cloud in the Cern SPS", Proceedings of IPAC'10, Kyoto, Japan (2010).

[37] L. Wang and M.T.F. Pivi, "Electron Trapping in Wiggler and Quadrupole Magnets of CesrTA", Proceedings of IPAC"10, Kyoto, Japan (2010).

[38] J.P. Shanks, et al., "CesrTA Low Emittance Tuning", Proceedings of IPAC"10, Kyoto, Japan (2010).

[39] D.P. Peterson, et al., "CesrTA X-Ray Beam Size Monitor Operation”, Proceedings of IPAC'10, Kyoto, Japan (2010).

[40] J. Crittenden, et al., "Studies of the Effects of Electron Cloud Formation on Beam Dynamics at CesrTA", Proceedings of PAC09, Vancouver, British Columbia, CA (2009).

[41] J. Crittenden, et al., "Progress in Studies of Electron Cloud -Induced Optics Distortions at CesrTA", Proceedings of IPAC'10, Kyoto, Japan (2010).

[42] G. .Dugan, et al., "Synrad3D Photon Propagation and Scattering Simulation", to appear in the Proceedings of the ECLOUD10 Workshop, Ithaca, NY, USA (2010).

[43] G. Dugan, et al., "CesrTA EC-Induced Beam Dynamics", to appear in the Proceedings of the ECLOUD10 Workshop, Ithaca, NY, USA (2010).

[44] J.W. Flanagan, et al., "Measurement of Low-Emittance Beam with Coded Aperture X Ray Optics at CesrTA", Proceedings of IPAC"10, Kyoto, Japan (2010).

[45] J.W. Flanagan, et al., "xBSM Bunch-by-Bunch Measurements in EC Conditions at CesrTA", to appear in the Proceedings of the ECLOUD10 Workshop, Ithaca, NY, USA (2010).

[46] K. Ohmi, et al., "Electron Instability in Low Emittance Rings, CesrTA and SuperKEKB", to appear in the Proceedings of the ECLOUD10 Workshop, Ithaca, NY, USA (2010).

[47] M.T.F. Pivi, et al., "ILC Damping Ring Electron Cloud R\&D Effort and Single-Bunch Instability Simulations Using CMAD", to appear in the Proceedings of the ECLOUD10 Workshop, Ithaca, NY, USA (2010).

[48] F. Zimmermann and G. Rumolo, Electron Cloud Effects in Accelerators, ICFA Beam Dynamics Newsletter No. 33, eds. K. Ohmi and M.A. Furman (2004)

[49] Wang, L. F. and Fukuma, H. and Ohmi, K. and Kurokawa, S. and Oide, K. and Zimmermann, F. "Numerical study of the photoelectron cloud in KEKB Low Energy Ring with a three-dimensional particle in cell method", Phys. Rev. STAB.5.124402, Dec. 2002

[50] M. A. Furman and G. R. Lambertson, Proc. MBI-97, KEK Proceedings 97-17, p. 170; M. A. Furman and M. T. F. Pivi, PRST-AB 5, 124404 (2002).

[51] J.-L.Vay et al., Update on Electron-Cloud Simulations Using the Package WARP/POSINST, Proc. of PAC09, FR5RFP078, p. 4719 (2009); http://www.JACoW.org. J.D. Fox et al., SPS Ecloud Instabilities - Analysis of Machine Studies and Implications for Ecloud Feedback, Proc. of IPAC10, Kyoto, Japan, WEPEB052, p. 2806 (2010).

[52] K. Ohmi and F. Zimmermann, Phys. Rev. Lett. 85, 3821 (2000)

[53] J. R. Calvey, J. A. Crittenden, G. Dugan, M.A.Palmer, C. M. Celata, "Electron Cloud Modeling Considerations at CesrTA," in Proceedings of the 2009 Particle Accelerator Conference, Vancouver, BC, 2009, p. 3306-3308. PDF (JACoW )

[54] B.L. Henke, E.M. Gullikson, and J.C. Davis. X-ray interactions: photoabsorption, scattering, transmission, and reflection at E=50-30000 eV, Z=1-92, Atomic Data and Nuclear Data Tables Vol. 54 (no.2), 181-342 


\section{international linear collider}

(July 1993). http://henke.lbl.gov/optical constants/

[55] M.T.Pivi, "CMAD: A New Self-consistent Parallel Code to Simulate the Electron Cloud Build-up and Instabilities", Proceedings 2007 Particle Accelerator Conference, Albuquerque, New Mexico

[56] K. G. Sonnad, K. Butler, G. Dugan, M. A. Palmer, M. T. F. Pivi, "Simulation of Electron Cloud Induced Instabilities and Emittance Growth for CesrTA," in Proceedings of the 2011 Particle Accelerator Conference, New York, NY, in press

[57] M. Palmer, et al., "CesrTA Preliminary Recommendations for the ILC Positron Damping Ring", to appear in the Proceedings of the ECLOUD10 Workshop, Ithaca, NY, USA (2010).

[58] M. Pivi, "Recommendation for the ILC DR EC mitigations“, slides 20-49, IWLC10 Workshop, CERN, October

2010 http://ilcagenda.linearcollider.org/materialDisplay.py?contribId=544\&sessionId=83\&materialId=slides\&confI $\underline{\mathrm{d}=4507}$ 